\title{
Unfoldings of complex analytic foliations with singularities
}

\author{
By Tatsuo SuwA*
}

(Received March 26, 1982)

In order to study the singularities of complex analytic foliations, we develop a theory of unfoldings in this paper. Section 1 contains terminologies and definitions on foliations with singularities. In section 2, we introduce unfoldings of foliations and define the infinitesimal unfolding map using some homological algebra. In section 3, we give explicit descriptions of the objects that appear in section 2 for later use.

In section 4 , we consider first order unfoldings of a local foliation $\boldsymbol{F}$ and give an embedding of the set $U(F)$ of equivalence classes of first order unfoldings of $\boldsymbol{F}$ into the group $\operatorname{Ext}_{o}^{1}\left(\Omega_{F}, \mathcal{O}\right)$ (cf. (4.5)). In general, $U(F)$ is smaller than $\operatorname{Ext}_{0}^{1}\left(\Omega_{F}, \mathcal{O}\right)$ and we may say that the difference of $U(F)$ from $\operatorname{Ext}_{\odot}^{1}\left(\Omega_{F}, \mathcal{O}\right)$ is the primary obstruction to finding integrating factors for a system of generators of $\boldsymbol{F}$ (cf. section 6).

We associate, in section 5, a local cohomology class to a 2-form satisfying a certain condition (cf. (5.2)). This is used to describe the set $U(F)$ in section 6 ((6.1) Theorem). Then we give an algorithm to find the first order unfoldings of a given local foliation (also a simpler one if it is of codimension 1). This together with a versality theorem in [10] enables us to construct universal unfoldings of some foliation singularities. Finally in section 7, we determine the set of equivalence classes of first order unfoldings of a global foliation.

A part of the contents of this paper is summarized in [13].

\section{§1. Complex analytic foliations with singularities}

In this section we give terminologies and definitions on complex analytic foliations with singularities (cf. Baum [1], Baum-Bott [2], see also Thom [16], [17]).

Let $M$ be a connected complex manifold of dimension $n$ and let $\mathcal{O}_{M}, \Omega_{M}^{p}$ and $\Theta_{M}$ denote, respectively, the sheaves of germs of holomorphic functions, holomorphic $p$-forms and holomorphic vector fields on $M$. The sheaf $\Omega_{M}^{1}$

*) Partially supported by the National Science Foundation. 
will be denoted simply by $\Omega_{M}$. For each point $z$ in $M$, let $\Omega_{M, z}^{p}$ denote the stalk of $\Omega_{M}^{p}$ at $z$. The exterior product and the exterior differentiation make the direct sum $\Lambda_{z}=\bigoplus_{p=0}^{n} \Omega_{M, z}^{p}\left(\Omega_{M}^{0}=\mathcal{O}_{M}\right)$ a differential graded $\mathcal{O}_{M, z}$-algebra. If $F$ is a subsheaf of $\Omega_{M}$, we denote by $\left(\Omega_{M} \wedge F\right)_{z}$ the degree 2 component of the ideal in $\Lambda_{z}$ generated by $F_{z}$.

For a coherent sub- $\mathcal{O}_{M}$-module $F$ of $\Omega_{M}$, the quotient sheaf $\Omega_{M} / F$ is denoted by $\Omega_{F}$ and the singular set $S(F)$ of $F$ is defined to be the singular set of the coherent sheaf $\Omega_{F}$;

$$
S(F)=\left\{z \in M \mid \Omega_{F, z} \text { is not a free } \mathcal{O}_{M, z} \text {-module }\right\} .
$$

If $\omega_{i}=\sum_{j=1}^{n} f_{i j}(z) d z_{j}, 1 \leq i \leq r$, denote generators of $F$ on a coordinate neighborhood $U$ in $M$ with coordinates $\left(z_{1}, z_{2}, \cdots, z_{n}\right)$, then $S(F) \cap U$ is the set of points $z$ in $U$ where the rank of the matrix $\left(f_{i j}(z)\right)_{1 \leq i \leq r, 1 \leq j \leq n}$ fails to be maximal. Thus $S(F)$ is an analytic set of $M$. Also, the annihilator $F^{a}$ of $F$ is the sub- $\mathcal{O}_{M}$-module of $\Theta_{M}$ defined by the presheaf

$$
U \longmapsto\left\{\theta \in \Gamma\left(U, \Theta_{M}\right) \mid \omega(\theta)=0 \quad \text { for all } \omega \in \Gamma(U, F)\right\},
$$

where $\Gamma(U, \quad)$ denotes the space of sections over $U$. There is a canonical isomorphism between $F^{a}$ and the sheaf of germs of $\mathcal{O}_{M}$-homomorphisms of $\Omega_{F}$ into $\mathcal{O}_{M}$;

$$
F^{a} \simeq \operatorname{Hom}_{\text {OHI }}\left(\Omega_{F}, \mathcal{O}_{M}\right) .
$$

The annihilator $\left(F^{a}\right)^{a}$ of $F^{a}$ is defined dually and is a sub- $\mathcal{O}_{M}$-module of $\Omega_{M}$. Note that $F$ is included in $\left(F^{a}\right)^{a}$. The subsheaf $F$ is said to be full in $\Omega_{M}$ if for any open set $U$ of $M$ and for any $\omega$ in $\Gamma\left(U, \Omega_{M}\right), \omega \in \Gamma(U-S(F), F)$ implies $\omega \in \Gamma(U, F)$. The sheaves $F^{a}$ and $\left(F^{a}\right)^{a}$ are full, respectively, in $\Theta_{M}$ and $\Omega_{M}$. It is easily shown that $F$ is full if and only if $F=\left(F^{a}\right)^{a}$. In general, $S(F)$ contains the singular set $S\left(F^{a}\right)$,

$$
S\left(F^{a}\right)=\left\{z \in M \mid\left(\Theta_{M} / F^{a}\right)_{z} \text { is not a free } \mathcal{O}_{M, z} \text {-module }\right\},
$$

of $F^{a}$. Hence if $F$ is full, then we have $S(F)=S\left(F^{a}\right)$.

(1.2) Definition. A foliation (with singularities) on $M$ is a coherent sub- $\mathcal{O}_{M}$-module $F$ of $\Omega_{M}$ which is integrable in the sense that

$$
d F_{z} \subset\left(\Omega_{M} \wedge F\right)_{z} \quad \text { for all } z \text { in } M-S(F) .
$$

A foliation $F$ is reduced if it is a full subsheaf of $\Omega_{M}$.

Note that the above integrability condition is weaker than [1] Definition 1.2 (ii). 
If $(M, F)$ is a foliation, then by the Frobenius theorem, $F$ defines an ordinary complex analytic foliation (without singularity) on $M-S(F)$. Its codimension is called the codimension of $F$. We call the quotient sheaf $\Omega_{F}=$ $\Omega_{M} / F$ the sheaf of germs of holomorphic 1-forms along the leaves of $F$. We record the exact sequence for later use:

$$
0 \longrightarrow F \stackrel{\iota}{\longrightarrow} \Omega_{M} \stackrel{\eta}{\longrightarrow} \Omega_{F} \longrightarrow 0 .
$$

Let $E$ be a coherent sub- $\mathcal{O}_{M}$-module of $\Theta_{M}$. We say that $E$ is integrable if

$$
\left[E_{z}, E_{z}\right] \subset E_{z}
$$

holds for all $z$ in $M-S(E)$. Note that if moreover $E$ is full in $\Theta_{M}$, then (1.4) holds for all $z$ in $M$.

The proof of the following is straightforward.

(1.5) Proposition. (i) If $(M, F)$ is a foliation, then $F^{a}$ is a coherent full sub- $\mathcal{O}_{M}$-module of $\Theta_{M}$ which is integrable.

(ii) If $(M, F)$ is a foliation, then $\left(M,\left(F^{a}\right)^{a}\right)$ is a reduced foliation.

(iii) There is a canonical one-to-one correspondence between the set of reduced foliations on $M$ and the set of coherent sub- $\mathcal{O}_{M}$-modules of $\Theta_{M}$ which are full and integrable.

For a foliation $F$ on $M$, we call $\left(F^{a}\right)^{a}$ the reduction of $F$.

(1.6) ExAmple. Let $M$ be the 2-dimensional complex affine space $C^{2}$ with coordinates $\left(z_{1}, z_{2}\right)$ and let $F$ be generated by the 1-form $\omega=z_{1} z_{2} d z_{1}$ $z_{1}^{2} d z_{2}$. Then $F^{a}$ is generated by $\theta=z_{1}\left(\partial / \partial z_{1}\right)+z_{2}\left(\partial / \partial z_{2}\right)$ and the reduction of $F$ is generated by $\omega_{0}=z_{2} d z_{1}-z_{1} d z_{2}$. We have $S(F)=\left\{z_{1}=0\right\}$, whereas $S\left(F^{a}\right)=$ $S\left(\left(F^{a}\right)^{a}\right)=\{(0,0)\}$.

Let $f: M^{\prime} \rightarrow M$ be a holomorphic map of a complex manifold $M^{\prime}$ into another $M$ and let $F$ be a sub- $\mathcal{O}_{M}$-module of $\Omega_{M}$. For each open set $U^{\prime}$ of $M^{\prime}$, we denote by $f^{* *} F\left(U^{\prime}\right)$ the sub- $\Gamma\left(U^{\prime}, \mathcal{O}_{M^{\prime}}\right)$-module of $\Gamma\left(U^{\prime}, \Omega_{M^{\prime}}\right)$ generated by the set

$$
\left\{f^{*} \omega \mid \omega \in \Gamma\left(f\left(U^{\prime}\right), F\right)\right\},
$$

where $f^{*} \omega$ denotes the pull-back of the 1-form $\omega$ by $f$. Note that each section $\omega$ in $\Gamma\left(f\left(U^{\prime}\right), F\right)$ can be extended to a 1-form on an open set containing $f\left(U^{\prime}\right)$.

(1.7) Definition. The pull-back $f^{* *} F$ of a sub- $\mathcal{O}_{M}$-module $F$ of $\Omega_{M}$ by a holomorphic map $f: M^{\prime} \rightarrow M$ is the sub- $\mathcal{O}_{M^{\prime}}$-module of $\Omega_{M^{\prime}}$ determined by the presheaf $U^{\prime} \mapsto f^{* *} F\left(U^{\prime}\right)$. Especially, if $M^{\prime}$ is a closed submanifold of $M$ and 
if $f$ is the inclusion, then the pull-back $f^{* *} F$ is also denoted by $F \|_{M^{\prime}}$ and is called the restriction of $F$ to $M^{\prime}$ as forms.

It is readily checked that $f^{* *} F$ is coherent.

(1.8) Remark. If $f: M^{\prime} \rightarrow M$ is a holomorphic map, then there is an exact sequence

$$
f^{*} \Omega_{M} \longrightarrow \Omega_{M^{\prime}} \longrightarrow \Omega_{M^{\prime} / M} \longrightarrow 0,
$$

where $f^{*} \Omega_{M}$ is the analytic inverse image of $\Omega_{M}$ by $f$ and $\Omega_{M^{\prime} / M}$ is the sheaf of germs of relative holomorphic 1-forms of $f$. The pull-back $f^{* *} \Omega_{M}$ coincides with the image of the homomorphism $f^{*} \Omega_{M} \rightarrow \Omega_{M^{\prime}}$. When the map $f$ is generically submersive, the homomorphism is injective. Thus in this case the analytic inverse image and the pull-back are identical.

(1.9) Definition. A foliation $(M, F)$ is a Haefliger foliation if each point of $M$ has a neighborhood on which $F$ is generated by exact forms.

(1.10) Definitions. A foliation $F$ is of complete intersection type if $F$ is a locally free $\mathcal{O}_{M}$-module.

Thus if $F$ is a codim $q$ foliation of complete intersection type, then each point of $M$ has a neighborhood $U$ on which $F$ is generated by $q$ 1-forms $\omega_{1}, \omega_{2}, \cdots, \omega_{q}$ such that $\omega_{1} \wedge \omega_{2} \wedge \cdots \wedge \omega_{q}(z) \neq 0$ for a general point $z$ in $U$. The singular set $S(F)$ is given by

$$
S(F) \cap U=\left\{z \in U \mid \omega_{1} \wedge \omega_{2} \wedge \cdots \wedge \omega_{q}(z)=0\right\} .
$$

Also the integrability condition in (1.2) is given by

$$
d \omega_{i} \wedge \omega_{1} \wedge \omega_{2} \wedge \cdots \wedge \omega_{q}(z)=0, \quad 1 \leq i \leq q
$$

for all $z$ in $U$.

If $F$ is a foliation of complete intersection type, then there is an associated vector bundle. However, in general, the bundle cannot be embedded into the holomorphic cotangent bundle of $M$ in such a way that the embedding induces the inclusion $F G \Omega_{M}$.

In what follows, we only deal with foliations of complete intersection type unless stated otherwise.

\section{§ 2. Unfoldings of foliations with singularities}

Let $F$ be a codim $q$ foliation (of complete intersection type) on $M$. Consider an analytic family of deformations (simply, a deformation) 


$$
\pi: \mathscr{M} \longrightarrow T
$$

of $M$, i.e., $\pi$ is a holomorphic submersion of a complex manifold $\mathscr{M}$ onto another $T$ such that $\pi^{-1}(o)=M$ for some point $o$ of $T$. We assume that $\pi$ is proper when $M$ is compact. Denoting by $\Omega_{\mathscr{M} / T}$ the sheaf of germs of relative holomorphic 1-forms of $\pi$, we have the exact sequence

$$
0 \longrightarrow \pi^{*} \Omega_{T} \longrightarrow \Omega_{\mathscr{M}} \longrightarrow \Omega_{\mathscr{M} / T} \longrightarrow 0 .
$$

If we denote by $\mathscr{I}_{t}$ the ideal sheaf of $M_{t}=\pi^{-1}(t)$ in $\mathscr{M}$, then we have $\mathcal{O}_{M t}=$ $\mathcal{O}_{\mathscr{A}} \mid \mathscr{I}_{t}$. For an $\mathcal{O}_{\mathcal{M}}$-module $\mathscr{S}$, we set $\left.\mathscr{S}\right|_{M_{t}}=\mathscr{S} \otimes_{\mathcal{O}_{\mathscr{H}}} \mathcal{O}_{M_{t}}$. Since $\pi$ is a holomorphic submersion, the natural homomorphism

$$
\left.\left.\pi^{*} \Omega_{T}\right|_{M_{t}} \longrightarrow \Omega_{\mathscr{M}}\right|_{M_{t}}
$$

is injective. Also there is a canonical isomorphism

$$
\left.\Omega_{\mathscr{M} / T}\right|_{M t} \simeq \Omega_{M t} .
$$

Thus, from (2.1), we get the exact sequence

$$
\left.\left.0 \longrightarrow \pi^{*} \Omega_{T}\right|_{M_{t}} \longrightarrow \Omega_{\mathcal{H}^{\prime}}\right|_{M_{t}} \stackrel{\alpha_{t}}{\longrightarrow} \Omega_{M_{t}} \longrightarrow 0 .
$$

It is easy to show the following

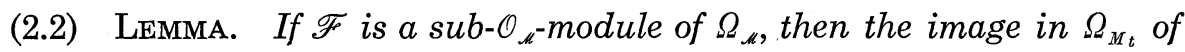
$\left.\mathscr{F}\right|_{M_{t}}$ by the composition of the canonical homomorphism $\left.\mathscr{F}_{M_{t} \rightarrow \Omega_{\mu l}}\right|_{M_{t}}$ and the homomorphism $\alpha_{t}$ in $(2.1)_{t}$ coincides with the restriction $\mathscr{F} \|_{M_{t}}$ of $\mathscr{F}$ to $M_{t}$ as forms ((1.7)).

(2.3) Definition. An unfolding of a codim $q$ foliation $(M, F)$ consists of (I) a deformation $\pi: \mathscr{M} \rightarrow T$ of $M$ (we let $\pi^{-1}(o)=M$ for $o \in T$ ) and

(II) a codim $q$ foliation $\mathscr{F}$ (of complete intersection type) on $\mathscr{M}$ such that $\mathscr{F} \|_{M}=F$.

(2.4) Remark. If $F$ is either a local foliation or a foliation on a compact $M$, then there exists a neighborhood $V$ of $o$ in $T$ such that $F_{t}=\mathscr{F} \|_{M_{t}}$ is a codim $q$ foliation (of complete intersection type) on $M_{t}$ for each $t$ in $V$. Thus $\left\{F_{t}\right\}_{t \in V}$ is an analytic family of deformations $F_{t}$ of $F$.

(2.5) Definition. An unfolding $\left(\mathscr{M}^{\prime} \stackrel{\pi^{\prime}}{\longrightarrow} T^{\prime}, \mathscr{F}^{\prime}\right)$ of $(M, F)$ is induced from another $(\mathscr{M} \rightarrow T, \mathscr{F})$ if there exist holomorphic maps $\Phi: \mathscr{M}^{\prime} \rightarrow \mathscr{M}$ and $\varphi: T^{\prime} \rightarrow T$ such that

(i) $\varphi$ maps the specific point $o^{\prime}$ in $T^{\prime}$ to the specific point $o$ in $T$,

(ii) $\varphi \circ \pi^{\prime}=\pi \circ \Phi$,

(iii) for each $t^{\prime}$ in $T^{\prime}, \Phi$ map $\pi^{\prime-1}\left(t^{\prime}\right)$ biholomorphically onto $\pi^{-1}\left(\varphi\left(t^{\prime}\right)\right)$, 
(iv) $\mathscr{F}^{\prime}=\Phi^{* *} \mathscr{F}((1.7))$.

An unfolding $(\mathscr{M} \stackrel{\pi}{\longrightarrow} T, \mathscr{F})$ of $(M, F)$ is versal if every unfolding of $F$ is induced from $\mathscr{F}$.

Let $(\mathscr{M} \stackrel{\pi}{\longrightarrow} T, \mathscr{F})$ be an unfolding of $(M, F)$. Then we have the diagram

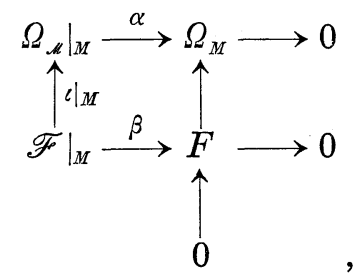

where $\iota$ denotes the inclusion $\mathscr{F} \subseteq \Omega_{M}, \alpha=\alpha_{o}\left((2.1)_{o}\right)$ and $\beta=\left.\alpha \circ \iota\right|_{M}$. By (2.3) (II), $\beta$ is surjective. Moreover, since both $\left.\mathscr{F}\right|_{M}$ and $F$ are locally free $\mathcal{O}_{M^{-}}$ modules of rank $q$, we see that $\beta$ is an isomorphism. Hence the homomorphism $\left.\iota\right|_{M}$ is injective. Setting $\Omega_{\mathscr{F}}=\Omega_{\mathcal{M}} / \mathscr{F}$, we obtain the commutative diagram with exact rows and columns:

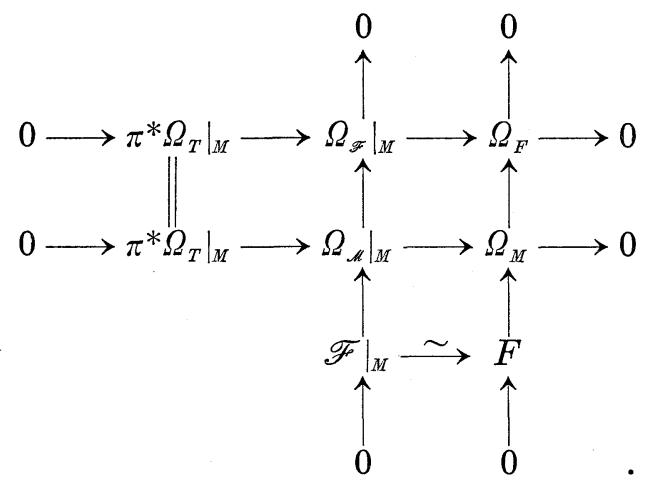

Letting $\mathscr{I}=\mathscr{I}_{0}$ be the ideal sheaf of $M$ in $\mathscr{M}$, there is a canonical isomorphism $\mathscr{F}\left|\mathscr{I} \mathscr{F} \simeq \mathscr{F} \otimes_{\mathscr{O}_{\mathcal{M}}} \mathcal{O}_{M}=\mathscr{F}\right|_{M}$. Hence there is a canonical surjection $\mathscr{F} \rightarrow F$. Let $\omega$ be a germ in $F_{z}$ for some $z$ in $M$. We call a germ $\tilde{\omega}$ in $\mathscr{F}_{z}$ an extension of $\omega$ if it is mapped to $\omega$ by the canonical surjection $\mathscr{F} \rightarrow F$. Now considering the exact sequence of the groups $\operatorname{Hom}_{\mathscr{O}_{M}}\left(\cdot, \mathcal{O}_{M}\right)$ of $\mathcal{O}_{M}$-module homomorphisms for the first row of (2.6), we have the connecting homomorphism

$$
\operatorname{Hom}_{\mathcal{O}_{M}}\left(\left.\pi^{*} \Omega_{T}\right|_{M}, \mathcal{O}_{M}\right) \longrightarrow \operatorname{Ext}_{\mathcal{O}_{M}}^{1}\left(\Omega_{F}, \mathcal{O}_{M}\right) \text {. }
$$

Denoting by $\pi^{*} \Theta_{T}$ the analytic inverse image of the sheaf $\Theta_{T}$ by $\pi$, we have the canonical isomorphisms 


$$
\operatorname{Hom}_{\mathcal{O}_{M}}\left(\left.\pi^{*} \Omega_{T}\right|_{M}, \mathcal{O}_{M}\right)=\Gamma\left(M, \mathscr{H}_{o m_{\mathcal{O}_{M}}}\left(\left.\pi^{*} \Omega_{T}\right|_{M}, \mathcal{O}_{M}\right)\right) \simeq \Gamma\left(M,\left.\pi^{*} \Theta_{T}\right|_{M}\right) .
$$

Let $T_{T, o}$ denote the holomorphic tangent space of $T$ at the specific point $o$ and let $T_{M}$ denote the constant sheaf $M \times T_{T, o}$ on $M$. Then the sheaf $T_{M}$ can be thought of as a subsheaf of $\left.\pi^{*} \Theta_{T}\right|_{M}$ in a canonical manner. We define the (first order) infinitesimal unfolding map

$$
\rho: T_{T, o} \longrightarrow \operatorname{Ext}_{O_{M}}^{1}\left(\Omega_{F}, \mathcal{O}_{M}\right)
$$

of the unfolding $(\mathscr{M} \stackrel{\pi}{\longrightarrow} T, \mathscr{F})$ at $o$ to be the composition of the inclusion $T_{T, o}=\Gamma\left(M, T_{M}\right) G_{H_{0}}\left(\left.\pi^{*} \Omega_{T}\right|_{M}, \mathcal{O}_{M}\right)$ and the homomorphism (2.7). We give an explicit description of the map (2.8) in the next section.

Now we consider the spectral sequence

$$
E_{2}^{p, q}=H^{p}\left(M, \mathscr{E}_{x t_{\mathcal{O}}}^{q}\left(\Omega_{F}, \mathcal{O}_{M}\right)\right) \Longrightarrow \operatorname{Ext}_{\mathscr{O}_{M}}^{n}\left(\Omega_{F}, \mathcal{O}_{M}\right) .
$$

Also, replacing $\Omega_{F}$ by $\Omega_{M}$, we have a similar spectral sequence, which degenerates since $\Omega_{M}$ is locally free. Noting that $\mathscr{E}_{x t_{O_{M}}^{0}}\left(\Omega_{F}, \mathcal{O}_{M}\right)=\mathscr{H}_{\text {om } \text { OH }_{M}}\left(\Omega_{F}, \mathcal{O}_{M}\right)$ $=F^{a}$, we get the commutative diagram with exact rows

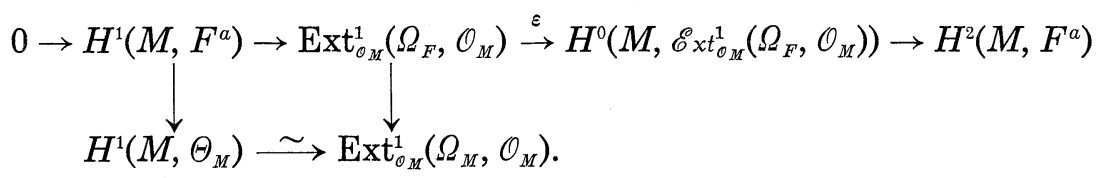

It is easily checked that the composition of (2.8) and the second vertical map is identical with the usual infinitesimal deformation map ([5] (6.2)) of the deformation $\pi: \mathscr{M} \rightarrow T$ at $o$ (cf. (3.7)). The sheaf $\mathscr{E}_{x t_{O_{M}}^{1}}\left(\Omega_{F}, \mathcal{O}_{M}\right)$ is supported on the singular set $S(F)$ of $F$ and contains some information about the singularity (see [12]).

\section{§3. The infinitesimal unfolding map}

In this section we give explicit descriptions of the spectral sequence (2.9) and the infinitesimal unfolding map (2.8).

Let $(M, F)$ be a codim $q$ foliation of complete intersection type and let

$$
\cdots \stackrel{d^{\prime \prime}}{\longrightarrow} \mathscr{L}_{q} \stackrel{d^{\prime \prime}}{\longrightarrow} \cdots \stackrel{d^{\prime \prime}}{\longrightarrow} \mathscr{L}_{1} \stackrel{d^{\prime \prime}}{\longrightarrow} \mathscr{L}_{0} \longrightarrow \Omega_{F} \longrightarrow 0
$$

be a resolution of $\Omega_{F}$ by locally free $\mathcal{O}_{M}$-modules $\mathscr{L}_{q}, q \geq 0$. In our case, we may actually set ((1.3))

$$
\mathscr{L}_{0}=\Omega_{M}, \mathscr{L}_{1}=F \quad \text { and } \quad \mathscr{L}_{q}=0 \text { for } q \geq 2 .
$$

We use this resolution hereafter. For an $\mathcal{O}_{M}$-module $\mathscr{S}$, we set $\mathscr{S}^{*}=$ 
$\mathscr{H}_{\mathrm{om}_{\mathcal{O}_{M}}}\left(\mathscr{S}, \mathcal{O}_{M}\right)$. If $\mathscr{U}=\left\{U_{\lambda}\right\}_{\lambda \in \Lambda}$ is an open covering of $M$, we denote by $C^{p}(\mathscr{U}, \mathscr{S})$ the group of $p$-cochains on $\mathscr{U}$ with coefficients in the sheaf $\mathscr{S}$. We have the coboundary map $d^{\prime}: C^{p}(\mathscr{U}, \mathscr{S}) \rightarrow C^{p+1}(\mathscr{U}, \mathscr{S})$. The homomorphism $\mathscr{L}_{q}^{*} \rightarrow \mathscr{L}_{q+1}^{*}$ induced from $d^{\prime \prime}$ is also denoted by $d^{\prime \prime}$. In the double complex

$$
C^{*}(\mathscr{U})=\left\{C^{p}\left(\mathscr{U}, \mathscr{L}_{q}^{*}\right) ; d^{\prime}, d^{\prime \prime}\right\}
$$

we have

$$
{ }^{\prime} H^{p \prime \prime} H^{q}\left(C^{\cdot \cdot}(\mathscr{U})\right)=H^{p}\left(\mathscr{U}, \mathscr{E} \times t_{\mathscr{O}_{M}}^{q}\left(\Omega_{F}, \mathcal{O}_{M}\right)\right) .
$$

Hence we see that the spectral sequence (2.9) is obtained by taking the inductive limit (as $\mathscr{U}$ runs through open coverings of $M$ ) of each term of the first spectral sequence

$$
{ }^{\prime} E_{2}^{p, q}={ }^{\prime} H^{p \prime \prime} H^{q}(C \cdot(\mathscr{U})) \Longrightarrow H^{n}\left(C^{\cdot *}(\mathscr{U})\right)
$$

associated with the double complex $C \cdot(\mathscr{U})$. Since the $\mathcal{O}_{M}$-modules $\mathscr{E} x t_{\mathscr{O}}^{q}\left(\Omega_{F}, \mathcal{O}_{M}\right)$ are coherent for all $q \geq 0$, if $\mathscr{U}$ is a Stein covering of $M$, then the spectral sequence (3.1) itself can be identified with (2.9). Using (1.1), from (1.3), we get the exact sequence

$$
0 \longrightarrow F^{a} \longrightarrow \Theta_{M} \stackrel{\iota^{*}}{\longrightarrow} F^{*} \longrightarrow \mathscr{E}^{*} \times t_{\mathscr{O}_{M}}^{1}\left(\Omega_{F}, \mathcal{O}_{M}\right) \longrightarrow 0 .
$$

Let $\mathscr{U}=\left\{\mathscr{U}_{\lambda}\right\}_{\lambda \in A}$ be a covering of $M$ by small polydisks. We denote the group $C^{p, q}(\mathscr{U})$ simply by $C^{p, q}$ and we set $d=d^{\prime}+(-1)^{p} d^{\prime \prime}: C^{p, q} \rightarrow C^{p+1, q}+$ $C^{p, q+1}$. Now we examine the groups $H^{1}\left(C^{\cdot}\right)=\operatorname{Ext}_{\mathscr{O}_{M}}^{1}\left(\Omega_{F}, \mathcal{O}_{M}\right),{ }^{\prime} E_{2}^{1,0}=H^{1}\left(M, F^{a}\right)$ and $\quad E_{2}^{0,1}=H\left(M, \mathscr{E}_{x t_{\mathscr{O}}^{1}}^{1}\left(\Omega_{F}, \mathcal{O}_{M}\right)\right)$. First we have

$$
\operatorname{Ext}_{\mathscr{O}_{M}}^{1}\left(\Omega_{F}, \mathcal{O}_{M}\right)=H^{1}\left(C^{\cdot *}\right)=\frac{\left\{c \in C^{1,0}+C^{0,1} \mid d c=0\right\}}{d C^{0,0}} .
$$

Take an element $c$ in $C^{1,0}+C^{0,1}$ and write $c=c_{1}+c_{2}$, where $c_{1}$ is in $C^{1,0}=$ $C^{1}\left(\mathscr{U}, \mathscr{L}_{0}^{*}\right)=C^{1}\left(\mathscr{U}, \Omega_{M}^{*}\right)=C^{1}\left(\mathscr{U}, \Theta_{M}\right)$ and $c_{2}$ is in $C^{0,1}=C^{0}\left(\mathscr{U}, \mathscr{L}_{1}^{*}\right)=C^{0}\left(\mathscr{U}, F^{*}\right)$. We have $d c=d^{\prime} c_{1}-d^{\prime \prime} c_{1}+d^{\prime} c_{2}+d^{\prime \prime} c_{2}$. Since the elements $d^{\prime} c_{1},-d^{\prime \prime} c_{1}+d^{\prime} c_{2}$ and $d^{\prime \prime} c_{2}$ are, respectively, in $C^{2,0}, C^{1,1}$ and $C^{0,2}$, the identity $d c=0$ is equivalent to

$$
\begin{array}{lll}
d^{\prime} c_{1}=0 & \text { in } & C^{2,0}=C^{2}\left(\mathscr{U}, \Theta_{M}\right), \\
d^{\prime \prime} c_{1}=d^{\prime} c_{2} & \text { in } & C^{1,1}=C^{1}\left(\mathscr{U}, F^{*}\right) \\
d^{\prime \prime} c_{2}=0 & \text { in } & C^{0,2}=C^{0}\left(\mathscr{U}, \mathscr{L}_{2}^{*}\right)=0 .
\end{array}
$$

We may write $d^{\prime \prime} c_{1}=\iota^{*} c_{1}((3.2))$. Setting $\theta=c_{1}, \varphi=c_{2}$ and $\delta=d^{\prime}$, we obtain the 
expression

$$
\operatorname{Ext}_{\mathscr{O}_{M}}^{1}\left(\Omega_{F}, \mathcal{O}_{M}\right)=\frac{\left\{(\theta, \varphi) \in Z^{1}\left(\mathscr{U}, \Theta_{M}\right) \times C^{0}\left(\mathscr{U}, F^{*}\right) \mid \iota^{*} \theta=\delta \varphi\right\}}{\left\{\left(\delta c, \iota^{*} c\right) \mid c \in C^{0}\left(\mathscr{U}, \Theta_{M}\right)\right\}},
$$

where $Z^{1}\left(\mathscr{U}, \Theta_{M}\right)$ denotes the group of 1 -cocycles on $\mathscr{U}$ with coefficients in $\Theta_{M}$. Similarly we have

$$
H^{1}\left(M, F^{a}\right)=\frac{Z^{1}\left(\mathscr{U}, F^{a}\right)}{\delta C^{0}\left(\mathscr{U}, F^{a}\right)} .
$$

The edge homomorphism $H^{1}\left(M, F^{a}\right) \rightarrow \operatorname{Ext}_{\mathscr{O}_{M}}^{1}\left(\Omega_{F}, \mathcal{O}_{M}\right)$ in (2.10) sends the class of a cocycle $\theta$ in $Z^{1}\left(\mathscr{U}, F^{a}\right)$ to the class of $(\theta, 0)$. Also we have

$$
H^{0}\left(M, \mathscr{E}_{x t_{O_{M}}^{1}}\left(\Omega_{F}, \mathcal{O}_{M}\right)\right)=\frac{\left\{(\theta, \varphi) \in C^{1}\left(\mathscr{U}, \Theta_{M}\right) \times C^{0}\left(\mathscr{U}, F^{*}\right) \mid \iota^{*} \theta=\delta \varphi\right\}}{\left\{\left(\delta c+c^{\prime}, \iota^{*} c\right) \mid c^{\prime} \in C^{1}\left(\mathscr{U}, F^{a}\right), c \in C^{0}\left(\mathscr{U}, \Theta_{M}\right)\right\}} .
$$

The edge homomorphism $\varepsilon$ in (2.10) sends the class of $(\theta, \varphi)$ to the class of $(\theta, \varphi)$.

Now we examine the infinitesimal unfolding map (2.8). Let $(\mathscr{M} \stackrel{\pi}{\rightarrow} T, \mathscr{F})$ be an unfolding of $(M, F)$ and let

$$
\mathscr{L} . \longrightarrow \Omega_{F} \longrightarrow 0{ }^{\prime} \mathscr{L} .\left.\longrightarrow \Omega_{\mathscr{F}}\right|_{M} \longrightarrow 0 \text { and } " \mathscr{L} .\left.\longrightarrow \pi^{*} \Omega_{T}\right|_{M} \longrightarrow 0
$$

be locally free resolutions such that we have the commutative diagram

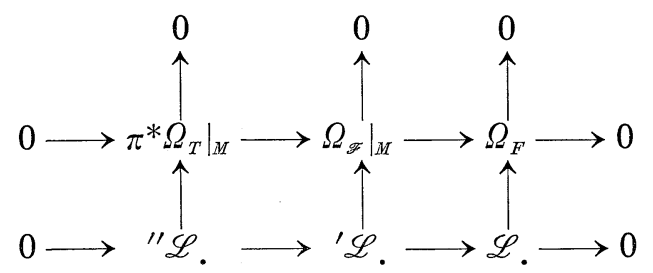

with exact rows (and columns). In our case we may actually set ((2.6))

$$
\begin{aligned}
\mathscr{L}_{0} & =\Omega_{M}, \quad \mathscr{L}_{1}=F, \quad \mathscr{L}_{q}=0 \text { for } q \geq 2, \\
{ }^{\prime} \mathscr{L}_{0} & =\left.\Omega_{\mu}\right|_{M}, \quad{ }^{2} \mathscr{L}_{1}=\left.\mathscr{F}\right|_{M}, \quad ' \mathscr{L}_{q}=0 \text { for } q \geq 2, \\
\prime \prime \mathscr{L}_{0} & =\left.\pi^{*} \Omega_{T}\right|_{M}, \quad " \quad \mathscr{L}_{q}=0 \text { for } q \geq 1 .
\end{aligned}
$$

We use these resolutions hereafter. Take a coordinate neighborhood $V$ on $T$ about the specific point $o$ with coordinates $t=\left(t_{1}, \cdots, t_{m}\right)$. Since $\pi$ is a submersion, $\pi^{-1}(V)$ has a covering $\left\{\mathscr{U}_{\lambda}\right\}_{\lambda \in A}$ by small polydisks $\mathscr{U}_{\lambda}$ such that each $\mathscr{U}_{\lambda}$ is a coordinate neighborhood on $\mathscr{M}$ with coordinates of the form $\left(z^{\lambda}, t\right)=\left(z_{1}^{\lambda}, \cdots, z_{n}^{\lambda}, t_{1}, \cdots, t_{m}\right), \pi\left(z^{\lambda}, t\right)=t$. In the intersection $\mathscr{U}_{\lambda} \cap \mathscr{U}_{\mu}, z^{\lambda}$ is a vector-valued holomorphic function of $\left(z^{\mu}, t\right)$; 


$$
z^{\lambda}=f^{\lambda \mu}\left(z^{\mu}, t\right)
$$

Let $\mathscr{U}=\left\{U_{\lambda}\right\}_{\lambda \in \Lambda}$ be the covering of $M$ defined by $U_{\lambda}=\mathscr{U}_{\lambda} \cap M$ and consider the double complexes

$$
C^{* *}=\left\{C^{p}\left(\mathscr{U}, \mathscr{L}_{q}^{*}\right)\right\},{ }^{\prime} C^{* *}=\left\{C^{p}\left(\mathscr{U}, \mathscr{L}_{q}^{*}\right)\right\} \quad \text { and } \quad{ }^{\prime \prime} C^{* *}=\left\{C^{p}\left(\mathscr{U},{ }^{\prime \prime} \mathscr{L}_{q}^{*}\right)\right\} .
$$

Denoting by $C^{*},{ }^{\prime} C^{*}$ and ${ }^{\prime \prime} C^{*}$, respectively, the total complexes of these double complexes, we get the exact sequence

$$
0 \longrightarrow C^{\cdot} \longrightarrow{ }^{\prime} C^{\cdot} \stackrel{\psi}{\longrightarrow}{ }^{\prime \prime} C^{\cdot} \longrightarrow 0 \text {. }
$$

The exact sequence of $\operatorname{Hom}_{\mathscr{O}_{M I}}\left(\cdot, \mathcal{O}_{M}\right)$ for the first row of (2.6) is identical with the cohomology exact sequence obtained from (3.6). Thus $\operatorname{Hom}_{\mathcal{O}_{M}}\left(\left.\pi^{*} \Omega_{T}\right|_{M}, \mathcal{O}_{M}\right)$ $=H^{0}\left({ }^{\prime \prime} C^{*}\right)=\left\{a \in{ }^{\prime \prime} C^{0} \mid d a=0\right\}$ and $\operatorname{Ext}_{\mathscr{O}_{M}}^{1}\left(\Omega_{F}, \mathcal{O}_{M}\right)=H^{1}\left(C^{*}\right)=\left\{c \in C^{1} \mid d c=0\right\} / d C^{0}$. Take a tangent vector $v=\sum_{k=1}^{m} c_{k}\left(\partial / \partial t_{k}\right)$ in $T_{T, o} \subset \operatorname{Hom}_{O_{M}}\left(\left.\pi^{*} \Omega_{T}\right|_{M}, \mathcal{O}_{M}\right)$ and choose an element $b$ in ${ }^{\prime} C^{0}=C^{0}\left(\mathscr{U}, \mathscr{H}_{\text {om }_{\mathcal{O}_{M}}}\left(\left.\Omega_{\mathscr{M}}\right|_{M}, \mathcal{O}_{M}\right)\right)=C^{0}\left(\mathscr{U},\left.\Theta_{\mathscr{M}}\right|_{M}\right)$ such that $\psi(b)=v$. We may write $b=\left\{b^{\wedge}\right\}$,

$$
b_{\lambda}=\sum_{k=1}^{m} c_{k} \frac{\partial}{\partial t_{k}} \quad \text { on } \quad U_{\lambda}
$$

We have $\psi d(b)=d \psi(b)=0$. Hence $d(b)$ is in (the image of) $C^{1}=C^{1,0}+C^{0,1}$. Write $d(b)=d^{\prime}(b)+d^{\prime \prime}(b)$ with $d^{\prime}(b)$ in $C^{1,0}=C^{1}\left(\mathscr{U}, \Theta_{M}\right)$ and $d^{\prime \prime}(b)$ in $C^{0,1}=$ $C^{0}\left(\mathscr{U}, \mathscr{H}_{\text {om }{ }_{O_{M}}}\left(F, \mathcal{O}_{M}\right)\right)$. If we $\operatorname{set} \theta=d^{\prime}(b), \theta$ is a collection $\left\{\theta^{\lambda \mu}\right\}$, where

$$
\begin{aligned}
\theta^{\lambda \mu}=b^{\mu}-b^{\lambda} & =\sum_{i=1}^{n} \sum_{k=1}^{m} c_{k} \frac{\partial z_{i}^{\lambda}}{\partial t_{k}} \frac{\partial}{\partial z_{i}^{\lambda}}+\sum_{j, k=1}^{m} c_{k} \frac{\partial t_{j}}{\partial t_{k}} \frac{\partial}{\partial t_{j}}-\sum_{k=1}^{m} c_{k} \frac{\partial}{\partial t_{k}} \\
& =\sum_{i=1}^{n} \sum_{k=1}^{m} c_{k} \frac{\partial f_{i}^{\lambda \mu}}{\partial t_{k}}\left(z^{\mu}, 0\right) \frac{\partial}{\partial z_{i}^{\lambda}} .
\end{aligned}
$$

Next we find $d^{\prime \prime}(b)$. Note that $d^{\prime \prime}(b)$ is a priori in ${ }^{\prime} C^{0,1}=C^{0}\left(\mathscr{U}, \mathscr{H}_{o m_{\mathcal{O}_{M}}}\left(\left.\mathscr{F}\right|_{M}, \mathcal{O}_{M}\right)\right)$ and that $d^{\prime \prime}$ is induced from the inclusion $\mathscr{F} \subset \Omega_{M}$. If we set $\varphi=d^{\prime \prime}(b), \varphi$ is a collection $\left\{\varphi^{\lambda}\right\}$, where, for each $\lambda, \varphi^{\lambda}$ is in $\Gamma\left(U_{\lambda}, \mathscr{H}_{\text {om } \mathcal{O}_{M}}\left(F, \mathcal{O}_{M}\right)\right)$ $\simeq \operatorname{Hom}_{\mathscr{O}_{M} \mid U_{\lambda}}\left(F\left|U_{\lambda}, \mathcal{O}_{M}\right|_{U_{\lambda}}\right)$. If $\omega=\sum_{i=1}^{n} f_{i}\left(z^{\lambda}\right) d z_{i}^{\lambda}$ is a 1 -form in $\Gamma\left(U_{\lambda}, F\right)$, then there is a 1 -form $\tilde{\omega}$ in $\Gamma\left(\mathscr{U}_{\lambda}, \mathscr{F}\right)$ which extends $\omega$. We may express

$$
\tilde{\omega}=\sum_{i=1}^{n} f_{i}\left(z^{\lambda}, t\right) d z_{i}^{\lambda}+\sum_{k=1}^{m} h_{k}\left(z^{\lambda}, t\right) d t_{k},
$$

where $f_{i}$ and $h_{k}$ are holomorphic functions on $\mathscr{U}_{\lambda}$ with $f_{i}\left(z^{\lambda}, 0\right)=f_{i}\left(z^{\lambda}\right)$. Although $\tilde{\omega}$ is not uniquely determined by $\omega$, the functions $h_{k}\left(z^{\lambda}, 0\right)$ on $U_{\lambda}$ are determined because of the isomorphism $\left.\mathscr{F}\right|_{M} \sim F$. Then we have 


$$
\varphi^{\lambda}(\omega)=\sum_{k=1}^{m} c_{k} h_{k}\left(z^{\lambda}, 0\right)
$$

In summary, the infinitesimal unfolding map (2.8) assigns to the vector $v=\sum_{k=1}^{m} c_{k}\left(\partial / \partial t_{k}\right)$ the class of $(\theta, \varphi)=\left(\left\{\theta^{\lambda \mu}\right\},\left\{\varphi^{\lambda}\right\}\right)((3.3))$ given by (3.7) and (3.8).

(3.9) Remark. Let $f: X \rightarrow Y$ be a holomorphic map of a compact complex manifold $X$ onto another $Y$. In this case the analytic inverse image $f^{*} \Omega_{Y}$ of $\Omega_{Y}$ by $f$ is identical with the pull-back $f^{* *} \Omega_{Y}((1.8))$. If we set $F=$ $f^{*} \Omega_{Y}$, comparing (3.3) and [4] Definition 4.1, we have

$$
\operatorname{Ext}_{\sigma_{X}}^{1}\left(\Omega_{F}, \mathcal{O}_{X}\right)=D_{X / Y} \text {. }
$$

We also have $\Omega_{F}=\Omega_{X / Y}$ and $F^{a}=\Theta_{X / Y}$ and the first sequence of (2.10) is identical with [4] (4.2).

\section{$\S 4$. First order unfoldings of local foliations}

We denote by ${ }_{n} \mathcal{O}$ or simply by $\mathcal{O}$ the local ring $\mathcal{O}_{C^{n}, 0}$ of germs of holomorphic functions at the origin 0 in $C^{n}=\left\{\left(z_{1}, \cdots, z_{n}\right)\right\}$, which is isomorphic to the ring $C\left\{z_{1}, \cdots, z_{n}\right\}$ of convergent power series in $\left(z_{1}, \cdots, z_{n}\right)$. We also denote the ${ }_{n} \mathcal{O}$-module $\Omega_{C^{n, 0}}$ by ${ }_{n} \Omega$. A local foliation $\boldsymbol{F}$ at 0 in $\boldsymbol{C}^{n}$ is defined to be the stalk $F_{0}$ at 0 of a foliation $F$ on a neighborhood $U$ of 0 in $C^{n} ; F=F_{0}$. If we denote the quotient $\mathcal{O}$-module ${ }_{n} \Omega / \boldsymbol{F}$ by $\Omega_{F}$, then this is identified with the stalk $\Omega_{F, 0}$ and we have the exact sequence

$$
0 \longrightarrow F \longrightarrow{ }_{n} \Omega \longrightarrow \Omega_{F} \longrightarrow 0
$$

of $\mathcal{O}$-modules. If $\boldsymbol{F}$ is a local foliation represented by $(U, F)$, then the singular set $S(F)$ of $\boldsymbol{F}$ is defined to be the germ at 0 of the singular set $S(F)$ of $F$. A local foliation $F$ is of complete intersection type if it is represented by a foliation of complete intersection type, in other words $F \simeq \mathcal{O}^{q}$ for some $q$. We only deal with local foliations of complete intersection type unless stated otherwise. Thus a codim $q$ local foliation at 0 in $C^{n}$ is a rank $q$ free sub- $\mathcal{O}$ module $\boldsymbol{F}=\left(\omega_{1}, \cdots, \omega_{q}\right)$ of ${ }_{n} \Omega$ such that $d \omega_{i} \wedge \omega_{1} \wedge \cdots \wedge \omega_{q}=0,1 \leq i \leq q$, where $\left(\omega_{1}, \cdots, \omega_{q}\right)$ is a system of generators of $\boldsymbol{F}$.

(4.2) Definition. Let $\boldsymbol{F}$ be a local foliation at 0 in $\boldsymbol{C}^{n}$. An unfolding $\mathscr{F}$ of $\boldsymbol{F}$ is a local foliation at 0 in $C^{n+m}$ represented by an unfolding ( $U \times V$

$\stackrel{\pi}{\longrightarrow} V, \mathscr{F})$ of $(U, F)$, where $(U, F)$ is a representative of $F, V$ is a neighborhood of 0 in $C^{m}$ and $\pi$ is the projection.

If $\mathscr{F}$ is an unfolding of a codim $q$ local foliation $\boldsymbol{F}$, then the infinitesimal unfolding map 


$$
\rho: T \longrightarrow \operatorname{Ext}_{0}^{1}\left(\Omega_{F}, \mathcal{O}\right),
$$

where $\boldsymbol{T}=\boldsymbol{T}_{C^{m, 0}}$, of $\mathscr{F}$ is defined in a similar manner as in section 2. From (4.1), we have a presentation of $\operatorname{Ext}_{o}^{1}\left(\Omega_{F}, \mathcal{O}\right)$;

$$
\cdots \longrightarrow \operatorname{Hom}_{0}\left({ }_{n} \Omega, \mathcal{O}\right) \longrightarrow \operatorname{Hom}_{0}(F, \mathcal{O}) \longrightarrow \operatorname{Ext}_{\odot}^{1}\left(\Omega_{F}, \mathcal{O}\right) \longrightarrow 0 .
$$

Let $\omega_{1}, \omega_{2}, \cdots, \omega_{q}$ be a system of generators of $\boldsymbol{F}$. We write

$$
\omega_{i}=\sum_{j=1}^{n} f_{i j} d z_{j}, \quad f_{i j} \in \mathcal{O}, \quad 1 \leq i \leq q .
$$

If we denote by $f_{j}$ the element $\left(f_{1 j}, \cdots, f_{q j}\right)$ in $\mathcal{O}^{q}$ and by $\left(f_{1}, \cdots, f_{n}\right)$ the sub- $\mathcal{O}$ module of $\mathscr{O}^{q}$ generated by $f_{1}, \cdots, f_{n}$, then we have, from (4.4),

$$
\operatorname{Ext}_{\odot}^{1}\left(\Omega_{F}, \mathcal{O}\right)=\mathcal{O}^{q} /\left(f_{1}, \cdots, f_{n}\right) .
$$

If $h=\left(h_{1}, \cdots, h_{q}\right)$ is an element in $\mathscr{O}^{q}$, we denote by $[h]=\left[h_{1}, \cdots, h_{q}\right]$ its image in $\operatorname{Ext}_{\sigma}^{1}\left(\Omega_{F}, \mathcal{O}\right)$ by the canonical surjection. For each $i, 1 \leq i \leq q$, let $\tilde{\omega}_{i}$ be an extension of $\omega_{i}$ in $\mathscr{F}$ and write $\tilde{\omega}_{i}=\sum_{j=1}^{n} f_{i j}(z, t) d z_{j}+\sum_{k=1}^{m} h_{i k}(z, t) d t_{k}$ in a neighborhood of 0 in $C^{n} \times C^{m}=\left\{\left(z_{1}, \cdots, z_{n} ; t_{1}, \cdots, t_{m}\right)\right\}$. Then the map (4.3) assigns to a tangent vector $\sum_{k=1}^{m} c_{k}\left(\partial / \partial t_{k}\right)$ in $T$ the element $\left[\sum_{k=1}^{m} c_{k} h_{1 k}, \cdots, \sum_{k=1}^{m} c_{k} h_{q k}\right]$ in $\operatorname{Ext}_{0}^{1}\left(\Omega_{F}, \mathcal{O}\right)$, where $h_{i k}$ is the germ at $z=0$ of the function $h_{i k}(z, 0)$ (see (3.8)).

Now let $U$ and $V$ be neighborhoods of the origins in $C^{n}=\left\{\left(z_{1}, \cdots, z_{n}\right)\right\}$ and $C=\{t\}$, respectively, and set $\mathscr{M}=U \times V$. The exterior product makes the direct $\operatorname{sum} \Lambda=\bigoplus_{p=0}^{n+1} H^{0}\left(\mathscr{M}, \Omega_{-\mu}^{p}\right)$ into a graded ring. Let $\left(t^{2}, t d t\right)$ denote the ideal in $\Lambda$ generated by $t^{2}$ and $t d t$. Two forms $\sigma$ and $\tau$ in $\Lambda$ are said to be identical up to the first order if $\sigma-\tau$ is in $\left(t^{2}, t d t\right)$. If this is the case, we write

$$
\sigma \equiv \tau \quad \bmod \left(t^{2}, t d t\right)
$$

(4.6) Definition. A first order unfolding of a codim $q$ foliation $(U, F)$ is a rank $q$ free sub- $\mathcal{O}_{U \times V}$-module $\mathscr{F}^{(1)}$ of $\Omega_{U \times V}$, for some neighborhood $V$ of 0 in $C$, such that

(i) $\mathscr{F} \mathscr{F}^{(1)} \|_{U \times 0}=F$ and

(ii) $\mathscr{F}^{(1)}$ satisfies the first order integrability condition, i.e., if $\left(\tilde{\omega}_{1}, \cdots\right.$, $\left.\tilde{\omega}_{q}\right)$ is a system of generators of $\mathscr{F}^{(1)}$ on $U \times V$, then

$$
d \tilde{\omega}_{i} \wedge \tilde{\omega}_{1} \wedge \cdots \wedge \tilde{\omega}_{q} \equiv 0 \quad \bmod \left(t^{2}, t d t\right) .
$$

A direct computation shows the following

(4.7) Lemma. Let $\left(\tilde{\omega}_{1}, \cdots, \tilde{\omega}_{q}\right)$ be a system of holomorphic 1-forms on $U \times V$. If 


$$
\omega_{i} \equiv \omega_{i}+\omega_{i}^{(1)} t+h_{i} d t \quad \bmod \left(t,{ }^{2} t d t\right), \quad 1 \leq i \leq q,
$$

where $\omega_{i}$ and $\omega_{i}^{(1)}$ are 1-forms involving only $z$ and $d z$ and $h_{i}$ are holomorphic functions of $z$, then the first order integrability condition (4.6) (ii) is given by

$$
\begin{aligned}
& d \omega_{i}^{(1)} \wedge \Omega-d \omega_{i} \wedge\left(\sum_{j=1}^{q}(-1)^{j} \omega_{j}^{(1)} \wedge \Omega_{j}\right)=0 \quad \text { and } \\
& \left(d h_{i}-\omega_{i}^{(1)}\right) \wedge \Omega+d \omega_{i} \wedge\left(\sum_{j=1}^{q}(-1)^{j} h_{j} \Omega_{j}\right)=0, \quad 1 \leq i \leq q,
\end{aligned}
$$

where $\Omega=\omega_{1} \wedge \cdots \wedge \omega_{q}$ and $\Omega_{j}=\omega_{1} \wedge \cdots \wedge \hat{\omega}_{j} \wedge \cdots \wedge \omega_{q}\left(\right.$ omit $\left.\omega_{j}\right)$.

(4.8) Remark. As is shown in the proof of (6.1) Theorem, the second equality above implies the first.

(4.9) Lemma. Let $\left(U \times V, \mathscr{F}{ }^{(1)}\right)$ be a first order unfolding of a codim $q$ foliation $(U, F)$ and let $\left(\omega_{1}, \cdots, \omega_{q}\right)$ be a system of generators of $F$ on $U$. Suppose $\left(\tilde{\omega}_{1}, \cdots, \tilde{\omega}_{q}\right)$ and $\left(\tilde{\omega}_{1}^{\prime}, \cdots, \tilde{\omega}_{q}^{\prime}\right)$ are two systems of generators of $\mathscr{F}^{(1)}$ extending the given system and write

$$
\begin{aligned}
& \tilde{\omega}_{i} \equiv \omega_{i}+\omega_{i}^{(1)} t+h_{i} d t, \\
& \tilde{\omega}_{i}^{\prime} \equiv \omega_{i}+\omega_{1}^{\prime(1)} t+h_{i}^{\prime} d t \quad \bmod \left(t^{2}, t d t\right)
\end{aligned}
$$

as in the previous lemma. Then $h_{i}=h_{i}^{\prime}$ for $i=1, \cdots, q$.

Proof. There exist holomorphic functions $f_{i j}, 1 \leq i, j \leq q$, on $U \times V$ such that $\tilde{\omega}_{1}^{\prime}=\sum_{j=1}^{q} f_{i j}(z, t) \omega_{j}$. If we write

$$
f_{i j}(z, t) \equiv f_{i j}(z)+f_{i j}^{(1)}(z) t \quad \bmod t^{2},
$$

then we see that $f_{i j}(z)=\delta_{i j}$. Hence we have $h_{i}^{\prime}=h_{i}$ for $i=1, \cdots, q$, Q.E.D.

(4.10) Definition. Two first order unfoldings $(U \times V, \mathscr{F}(1))$ and $\left(U \times V^{\prime}\right.$, $\left.\mathscr{F}^{\prime(1)}\right)$ of $(U, F)$ are equivalent if there is a biholomorphic map $\Phi$ of a neighborhood $N$ of $U \times 0$ in $U \times\left(V \cap V^{\prime}\right)$ into $U \times\left(V \cap V^{\prime}\right)$ such that

(i) $\pi \circ \Phi=\left.\pi\right|_{N}$, where $\pi$ is the projection $U \times\left(V \cap V^{\prime}\right) \rightarrow V \cap V^{\prime}$,

(ii) $\left.\Phi\right|_{U \times 0}=1_{U}$ (the identity map of $U$ ) and

(iii) $\left.\mathscr{F}^{\prime(1)}\right|_{N} \equiv \Phi^{* *}\left(\left.\mathscr{F}^{(1)}\right|_{\mathscr{W}(N)}\right) \quad \bmod \left(t^{2}, t d t\right)$.

(4.11) Definition. Let $\boldsymbol{F}$ be a local foliation at 0. A first order unfolding $\mathscr{F}^{(1)}$ of $\boldsymbol{F}$ is the stalk at 0 of a first order unfolding $\mathscr{F}^{(1)}$ of a representative $F$ of $\boldsymbol{F}$. Two first order unfoldings $\mathscr{F}^{(1)}$ and $\mathscr{F}^{\prime(1)}$ of $\boldsymbol{F}$ are equivalent if they are represented by equivalent first order unfoldings of a representative of $F$.

Now let $\boldsymbol{F}$ be a codim $q$ local foliation and fix a system $\left(\omega_{1}, \cdots, \omega_{q}\right)$ of 
generators of $\boldsymbol{F}$. Then, by (4.9), each first order unfolding $\mathscr{F}^{(1)}$ of $\boldsymbol{F}$ determines a system of germs of functions $\left(h_{1}, \cdots, h_{q}\right)$.

(4.12) Lemma. Let $\mathscr{F}^{(1)}$ and $\mathscr{F}^{\prime(1)}$ be two first order unfoldings of $\boldsymbol{F}$ and let $\left(h_{1}, \cdots, h_{q}\right)$ and $\left(h_{1}^{\prime}, \cdots, h_{q}^{\prime}\right)$ be the systems of germs determined by $\mathscr{F}^{(1)}$ and $\mathscr{F}^{\prime(1)}$, respectively. Then $\mathscr{F}^{(1)}$ and $\mathscr{F}^{\prime(1)}$ are equivalent if and only if

$$
\left[h_{1}, \cdots, h_{q}\right]=\left[h_{1}^{\prime}, \cdots, h_{q}^{\prime}\right] \quad \text { in } \operatorname{Ext}_{\odot}^{1}\left(\Omega_{F}, \mathcal{O}\right) .
$$

Proof. Let $\left(\tilde{\omega}_{1}, \cdots, \tilde{\omega}_{q}\right)$ and $\left(\tilde{\omega}_{1}^{\prime}, \cdots, \tilde{\omega}_{q}^{\prime}\right)$ be systems of generators of $\mathscr{F}^{(1)}$ and $\mathscr{F}^{\prime(1)}$, respectively, extending the given system $\left(\omega_{1}, \cdots, \omega_{q}\right)$. We write

$$
\begin{aligned}
& \tilde{\omega}_{i} \equiv \omega_{i}+\omega_{i}^{(1)} t+h_{i} d t, \\
& \tilde{\omega}_{i}^{\prime} \equiv \omega_{i}+\omega_{i}^{\prime(1)} t+h_{i}^{\prime} d t \quad \bmod \left(t^{2}, t d t\right) .
\end{aligned}
$$

Suppose $\mathscr{F}^{(1)}$ and $\mathscr{F}^{\prime(1)}$ are equivalent and let $\Phi$ be a biholomorphic map as in (4.10). If we write $\Phi(z, t)=(\varphi(z, t), t)$ and $\varphi(z, t) \equiv z+\varphi^{(1)}(z) t \bmod \left(t^{2}\right)$, then we have

$$
\Phi^{*} \tilde{\omega}_{i} \equiv \omega_{i}+\omega_{i}^{\prime \prime(1)} t+\left(h_{i}+\sum_{j=1}^{n} f_{i j} \varphi_{j}^{(1)}\right) d t \quad \bmod \left(t^{2}, t d t\right)
$$

where $\varphi_{j}^{(1)}$ is the $j$-th component of $\varphi^{(1)}$ and $f_{i j}$ are coefficients of $\omega_{i} ; \omega_{i}=$ $\sum_{j=1}^{n} f_{i j}(z) d z_{j}, 1 \leq i \leq q$. Hence, by (4.9) Lemma, we have

$$
h_{i}^{\prime}=h_{i}+\sum_{j=1}^{n} f_{i j} \varphi_{j}^{(1)}, \quad 1 \leq i \leq q .
$$

This shows that $\left[h_{1}^{\prime}, \cdots, h_{q}^{\prime}\right]$ and $\left[h_{1}, \cdots, h_{q}\right]$ are identical in $\operatorname{Ext}_{\odot}^{1}\left(\Omega_{F}, \mathcal{O}\right)$. Conversely, suppose $\left[h_{1}^{\prime}, \cdots, h_{q}^{\prime}\right]=\left[h_{1}, \cdots, h_{q}\right]$. Then there are holomorphic functions $\varphi_{j}^{(1)}(z), 1 \leq j \leq n$, such that (4.13) holds. Let $\Phi$ be defined by $\Phi(z, t)$ $=\left(z+\varphi^{(1)}(z) t, t\right)$. Then it is easily shown that $\Phi$ satisfies the conditions of (4.10),

Q.E.D.

We denote by $U(F)$ the set of equivalence classes of first order unfoldings of $\boldsymbol{F}$. Then by the above lemma, there is an embedding

$$
U(F) \subseteq \operatorname{Ext}_{\odot}^{1}\left(\Omega_{F}, \mathcal{O}\right),
$$

which assigns $\left[h_{1}, \cdots, h_{q}\right]$ to the equivalence class of $\mathscr{F}^{(1)}$.

$\S 5$. Certain local cohomology class associated with a 2-form $\tau$ satisfying $\tau \wedge \omega_{1} \wedge \cdots \wedge \omega_{q}=0$

Let $\boldsymbol{F}$ be a codim $q$ local foliation at 0 in $\boldsymbol{C}^{n}$ and let $S(\boldsymbol{F})$ be the singular 
set of $\boldsymbol{F}$. We say that $\boldsymbol{F}$ is reduced if it is represented by a reduced foliation. First we prove the following

(5.1) Lemma. The codimension of the germ $S(F)$ of analytic set at 0 in $\boldsymbol{C}^{n}$ is greater than one if and only if $\boldsymbol{F}$ is reduced.

Proof. We let $\boldsymbol{F}$ be represented by a foliation $F$ on a small neighborhood $U$ of 0 in $C^{n}$ and take a system $\left(\omega_{1}, \cdots, \omega_{q}\right)$ of generators of $F$ on $U$. Suppose codim $S(F) \geq 2$. Then the Hartogs' continuation theorem shows that $F$ is reduced. We now suppose that codim $S(F)=1$. Then there is a germ $f$ in the ring $\mathcal{O}$ such that every germ in the ideal of $S(F)$ is divisible by $f$ in $\mathcal{O}$. We write $\omega_{i}=\sum_{j=1}^{n} f_{i j}(z) d z_{j}, 1 \leq i \leq q$, where $f_{i j}$ are holomorphic functions on $U$. Let $f\left(i_{1}, \cdots, i_{r} ; j_{1}, \cdots, j_{r}\right)$ denote the $r \times r$ minor

$$
\left|\begin{array}{ccc}
f_{i_{1} j_{1}} & \cdots & f_{i_{1} j_{r}} \\
\vdots & & \vdots \\
f_{i_{r} j_{1}} & \cdots & f_{i_{r} j_{r}}
\end{array}\right|
$$

of the $q \times n$ matrix $\left(f_{i j}\right)$. If all the $f_{i j}$ are divisible by $f$, then, for example, the form $\omega_{1} / f$ is holomorphic on $U$ and is in $\Gamma(U-S(F), F)$ but not in $\Gamma(U, F)$. Hence $F$ is not reduced. From now on we assume that there is an $f_{i j}$ which is not divisible by $f$. By (1.12), $S(F)$ is the set of common zeros of the functions

$$
f\left(1,2, \cdots, q ; j_{1}, j_{2}, \cdots, j_{q}\right), \quad 1 \leq j_{1}<j_{2}<\cdots<j_{q} \leq n .
$$

Hence all the $q \times q$ minors are divisible by $f$. Therefore, there exists a (unique) integer $p(2 \leq p \leq q)$, such that all the $p \times p$ minors of $\left(f_{i j}\right)$ are divisible by $f$ but there is a $(p-1) \times(p-1)$ minor which is not divisible by $f$. Without loss of generality, we may assume that the minor $f(1,2, \cdots, p-1$; $1,2, \cdots, p-1)$ is not divisible by $f$. Using the identities $\omega_{i}=\sum_{j=1}^{n} f_{i j} d z_{j}, 1 \leq i$ $\leq p$, we have

$$
\begin{aligned}
& \sum_{i=1}^{p}(-1)^{p+i} f(1, \cdots, \hat{i}, \cdots, p ; 1, \cdots, p-1) \omega_{i} \\
& =\sum_{j=p}^{n} f(1, \cdots, p ; 1, \cdots, p-1, j) d z_{j} .
\end{aligned}
$$

If we denote this form by $\omega$, then the 1 -form $\omega / f$ is holomorphic on $U$, since the functions $f(1, \cdots, p ; 1, \cdots, p-1, j)$ are divisible by $f$. The function (representing) $f$ does not vanish outside of $S(F)$. Hence $\omega / f$ is in $\Gamma(U-S(F), F)$. However, it is not in $\Gamma(U, F)$, since the function $f(1, \cdots$, $p-1 ; 1, \cdots, p-1)$ is not divisible by $f$. This shows that $F$ is not reduced. 
Now we fix a system $\left(\omega_{1}, \cdots, \omega_{q}\right)$ of generators of the germ $\boldsymbol{F}$. Let $\tau$ be a germ of holomorphic 2 -form at 0 in $C^{n}$ satisfying

$$
\tau \wedge \omega_{1} \wedge \cdots \wedge \omega_{q}=0
$$

We choose a small neighborhood $U_{0}$ of 0 so that the germs $\tau, \omega_{1}, \cdots, \omega_{q}$ have representatives on $U_{0}$. Let $F$ be the foliation on $U_{0}$ generated by $\omega_{1}, \cdots, \omega_{q}$ and set $S=S(F)$ and $S=S(F)$. Take a polydisk $U\left(\subset U_{0}\right)$ with center 0 and let $\left\{U_{\lambda}\right\}_{\lambda \in \Lambda}$ be an open covering of $U-S$ small polydisks $U_{\lambda}$. Since $\omega_{1}, \cdots, \omega_{q}$ are linearly independent at each point of $U-S$, from (5.2), $\tau$ may be written as

$$
\tau=\sum_{i=1}^{q} \eta_{i}^{\lambda} \wedge \omega_{i} \quad \text { on } U_{\lambda}
$$

where $\eta_{i}^{\lambda}, 1 \leq i \leq q$, are holomorphic 1 -forms on $U_{\lambda}$. In the intersection $U_{\lambda} \cap$ $U_{\mu}$, we have

$$
\sum_{i=1}^{q}\left(\eta_{i}^{\lambda}-\eta_{i}^{\mu}\right) \wedge \omega_{i}=0
$$

Hence we may write

$$
\eta_{i}^{\lambda}-\eta_{i}^{\mu}=\sum_{j=1}^{q} \psi_{i j}^{\lambda \mu} \omega_{j} \quad \text { on } U_{\lambda} \cap U_{\mu}
$$

where $\psi_{i j}^{\lambda \mu}$ are holomorphic functions on $U_{\lambda} \cap U_{\mu}$ satisfying

$$
\psi_{i j}^{\lambda \mu}=\psi_{j i}^{\lambda \mu}, \quad 1 \leq i, j \leq q,
$$

and for each $(i, j)$, the cocycle condition

$$
\psi_{i j}^{\lambda \mu}+\psi_{i j}^{\mu \nu}+\psi_{i j}^{\nu \lambda}=0 \quad \text { on } U_{\lambda} \cap U_{\mu} \cap U_{\nu} .
$$

If we set

$$
\Psi=\left\{\Psi^{\lambda \mu}\right\}, \quad \Psi^{\lambda \mu}=\left(\psi_{i j}^{\lambda \mu}\right)_{1 \leq i, j \leq q},
$$

then, by (5.6), $\Psi$ defines a cohomology class in $H^{1}\left(U-S, \mathcal{O}_{U}^{q^{2}}\right)$. It is not difficult to check that the cohomology class does not depend on the choice of $\eta_{i}^{\lambda}$ or $\psi_{i j}^{\lambda \mu}$ and is uniquely determined by the form $\tau$. We denote this cohomology class by $c_{U}(\tau)$. For our purpose, it is more convenient to think of $c_{U}(\tau)$ as a class in the cohomology $H_{S \cap U}^{2}\left(U, \mathcal{O}_{U}^{q^{2}}\right)$ with supports in $S \cap U$. In the local cohomology exact sequence

$$
\begin{aligned}
\cdots & \longrightarrow H^{1}\left(U, \mathcal{O}_{U}^{q^{2}}\right) \longrightarrow H^{1}\left(U-S, \mathcal{O}_{U}^{q^{2}}\right) \longrightarrow H_{S \cap U}^{2}\left(U, \mathcal{O}_{U}^{q^{2}}\right) \\
& \longrightarrow H^{2}\left(U, \mathcal{O}_{U}^{q^{2}}\right) \longrightarrow \cdots,
\end{aligned}
$$


we have $H^{p}\left(U, \mathscr{O}_{U}^{a^{2}}\right)=0$ for $p \geq 1$. Hence we have a canonical isomorphism

$$
H^{1}\left(U-S, \mathcal{O}_{U}^{q^{2}}\right) \simeq H_{S \cap U}^{2}\left(U, \mathcal{O}_{U}^{q^{2}}\right) .
$$

Thus we may think of $c_{U}(\tau)$ as a class in $H_{S \cap U}^{2}\left(U, \mathcal{O}_{U}^{q^{2}}\right)$. Let $\mathscr{H}_{S}^{p}\left(\mathcal{O}_{U_{0}}^{q^{2}}\right)$ denote the $p$-th cohomology sheaf (over $U_{0}$ ) with coefficients in $\mathcal{O}_{U_{0}}^{q^{2}}$ and supports in $S$. We denote by $\mathscr{H}_{S}^{p}\left(\mathcal{O}^{q 2}\right)$ the stalk $\mathscr{H}_{S}^{p}\left(\mathcal{O}_{U_{0}}^{q^{2}}\right)_{0}$. Then we have ([3] Proposition 1.2)

$$
\mathscr{H}_{S}^{p}\left(\mathcal{O}^{q^{2}}\right)=\underset{U}{\lim } H_{S \cap U}^{p}\left(U, \mathcal{O}_{U}^{q^{2}}\right),
$$

where $U$ runs through open neighborhoods of 0 in $U_{0}$. Clearly the image of $c_{U}(\tau)$ by the canonical homomorphism

$$
H_{S \cap U}^{2}\left(U, \mathcal{O}_{U}^{q^{2}}\right) \longrightarrow \mathscr{H}_{S}^{2}\left(\mathscr{O}^{q^{2}}\right)
$$

does not depend on the choice of $U$ and is uniquely determined by $\tau$. We denote the image by $c(\tau)$. We note that $\mathscr{H}_{S}^{2}\left(\mathcal{O}^{2}\right)=0$ if codim $S \geq 3$.

Next we examine the relation between the cohomology groups $\mathscr{H}_{S}^{2}\left(\mathcal{O}^{q^{2}}\right)$ and $H^{2}$, the latter being defined as

$$
\begin{aligned}
& Z^{2}=\left\{\tau \in \Omega_{C^{n, 0}}^{2} \mid \tau \wedge \omega_{1} \wedge \cdots \wedge \omega_{q}=0\right\}, \\
& B^{2}=\left\{\tau \in \Omega_{C^{n, 0},}^{2} \mid \tau=\sum_{i=1}^{q} \eta_{i} \wedge \omega_{i} \text { for some } \eta_{i} \text { in } \Omega_{c^{n, 0}}\right\}, \\
& H^{2}=Z^{2} / B^{2} .
\end{aligned}
$$

(5.8) Lemma. If codim $S \geq 2$, then there is a canonical embedding

$$
H^{2} G \mathscr{H}_{S}^{2}\left(\mathcal{O}^{q^{2}}\right) \text {. }
$$

Proof. We have a homomorphism

$$
Z^{2} \longrightarrow \mathscr{H}_{S}^{2}\left(\mathcal{O}^{q^{2}}\right)
$$

which assings to $\tau$ the cohomology class $c(\tau)$ defined as above. Suppose $c(\tau)=0$. Then there is a small polydisk $U$ with center 0 such that $\tau$ is represented by a 2 -form on $U$ and that $c_{U}(\tau)=0$ in $H_{S \cap U}^{2}\left(U, \mathcal{O}_{U}^{q^{2}}\right)$. Choose $\eta_{i}^{2}$ and $\psi_{i j}^{\lambda \mu}$ so that (5.3) and (5.4) hold. Since $c_{U}(\tau)=0$, we have, for each $\lambda$, a matrix-valued holomorphic function $\left(\varphi_{i j}^{\lambda}\right)_{1 \leq i, j \leq q}$ on $U_{\lambda}$ such that

$$
\psi_{i j}^{\lambda \mu}=\varphi_{i j}^{\mu}-\varphi_{i j}^{\lambda} \quad \text { on } U_{\lambda} \cap U_{\mu} .
$$

From (5.4), we have

$$
\eta_{i}^{2}+\sum_{j=1}^{q} \varphi_{i j}^{\lambda} \omega_{j}=\eta_{i}^{\mu}+\sum_{j=1}^{q} \varphi_{i j}^{\mu} \omega_{j} \quad \text { on } U_{\lambda} \cap U_{\mu} .
$$


This shows that we get, for each $i$, a holomorphic 1 -form $\xi_{i}$ on $U-S$ by setting $\xi_{i}=\eta_{i}^{\lambda}+\sum_{j=1}^{q} \varphi_{i j}^{\lambda} \omega_{j}$ on $U_{\lambda}$. By assumption, codim $S \geq 2$. Hence $\xi_{i}$ can be extended to a holomorphic 1-form on $U$, which we denote also by $\xi_{i}$. From (5.5), we have

$$
\varphi_{j i}^{\lambda}-\varphi_{i j}^{\lambda}=\varphi_{j i}^{\mu}-\varphi_{i j}^{\mu} \quad \text { on } U_{\lambda} \cap U_{\mu} .
$$

Hence we get a holomorphic function $f_{i j}$ on $U-S$ by setting $f_{i j}=\varphi_{j i}^{\lambda}-\varphi_{i j}^{\lambda}$ on $U_{\lambda}$. Again, each $f_{i j}$ can be extended to a holomorphic function on $U$. We then compute

$$
\begin{aligned}
\tau=\sum_{i=1}^{q} \eta_{i}^{\lambda} \wedge \omega_{i} & =\sum_{i=1}^{q} \xi_{i} \wedge \omega_{i}+\sum_{i, j=1}^{q} \varphi_{i j}^{\lambda} \omega_{j} \wedge \omega_{i} \\
& =\sum_{i=1}^{q} \xi_{i} \wedge \omega_{i}+\sum_{1 \leq i<j \leq q} f_{i j} \omega_{i} \wedge \omega_{j} .
\end{aligned}
$$

This shows that $\tau$ is in $B^{2}$. Hence the homomorphism (5.9) induces a monomorphism $H^{2} \rightarrow \mathscr{H}_{S}^{2}\left(\mathcal{O}^{q^{2}}\right)$.

Q.E.D.

(5.10) Corollary. If codim $S \geq 3$, then $H^{2}=0$.

(5.11) REMark. The above is a special case of the generalized de Rham lemma ([6] Proposition (2.1), [9] Theorem).

Now we take a system $\tau=\left(\tau_{1}, \cdots, \tau_{q}\right)$ of germs of holomorphic 2 -forms with each $\tau_{i}, 1 \leq i \leq q$, satisfying (5.2). Let $U$ be a small polydisk with center 0 on which $\omega_{1}, \cdots \omega_{q}, \tau_{1}, \cdots, \tau_{q}$ have representatives. We may write (see (5.3))

$$
\tau_{i}=\sum_{j=1}^{q} \eta_{i j}^{\lambda} \wedge \omega_{j}, \quad 1 \leq i \leq q, \quad \text { on } U_{\lambda}
$$

Then we have ((5.4) and (5.5))

$$
\begin{aligned}
\eta_{i j}^{\lambda}-\eta_{i j}^{\mu}= & \sum_{k=1}^{q} \psi_{i j k}^{\lambda \mu} \omega_{k}, \quad 1 \leq i, j \leq q, \quad \text { on } U_{\lambda} \cap U_{\mu}, \\
& \psi_{i j k}^{\lambda \mu}=\psi_{i k j}^{\lambda \mu}, \quad 1 \leq i, j, k \leq q .
\end{aligned}
$$

Let $h=\left(h_{1}, \cdots, h_{q}\right)$ be an element in $\mathcal{O}^{q}$. We may suppose, by taking a smaller $U$ if necessary, that each $h_{i}$ has a representative on $U$. We define $h c_{U}(\tau)$ to be the cohomology class in $H^{1}\left(U-S, \mathcal{O}_{U}^{q^{2}}\right) \simeq H_{S}^{2}\left(U, \mathcal{O}_{U}^{q^{2}}\right)$ represented by the cocycle

$$
\left\{\left(\sum_{j=1}^{q} h_{j} \psi_{i j k}^{\lambda \mu}\right)_{1 \leq i, k \leq q}\right\} .
$$


We denote by $h c(\tau)$ the image in $\mathscr{H}_{S}^{2}\left(\mathcal{O}^{q^{2}}\right)$ of $h c_{U}(\tau)$ by the homomorphism (5.7). It is easily checked that $h c(\tau)$ depends only on $h$ and $\tau$ and not on the representatives. Now we write

$$
\omega_{i}=\sum_{j=1}^{n} f_{i j} d z_{j}
$$

$1 \leq i \leq q$, and consider the systems $f_{k}=\left(f_{1 k}, \cdots, f_{q k}\right), 1 \leq k \leq n$. If we write $\eta_{i j}^{\lambda}=\sum_{k=1}^{q} g_{i j k}^{\lambda} d z_{k}$ on $U_{\lambda}$, then from (5.13) and (5.14) we have

$$
\sum_{l=1}^{q} f_{l k} \psi_{i i j}^{\lambda \mu}=\sum_{l=1}^{q} f_{l k} \psi_{i j l}^{\lambda \mu}=g_{i j k}^{\lambda}-g_{i j k}^{\mu} \quad \text { on } U_{\lambda} \cap U_{\mu} .
$$

This shows that $f_{k} c(\tau)=0$ in $\mathscr{H}_{S}^{2}\left(\mathcal{O}^{q 2}\right), 1 \leq k \leq n$. Therefore, for each element $[h]$ in $\operatorname{Ext}_{0}^{1}\left(\Omega_{F}, \mathcal{O}\right),[h] c(\tau)$ is a well defined element in $\mathscr{H}_{S}^{2}\left(\mathscr{O}^{q^{2}}\right)$.

(5.16) Remark. In this section we did not use the integrability of $\boldsymbol{F}$. We only need that $\boldsymbol{F}$ is a rank $q$ free sub-O-module of $\Omega_{\boldsymbol{C}^{n, 0}}$.

\section{§6. $U(F)$}

Let $\boldsymbol{F}$ be a codim $q$ local foliation at 0 in $\boldsymbol{C}^{n}$. We fix a system $\left(\omega_{1}, \cdots\right.$, $\omega_{q}$ ) of generators of $\boldsymbol{F}$ and set $d \omega=\left(d \omega_{1}, \cdots, d \omega_{q}\right)$. By the integrability

$$
d \omega_{i} \wedge \omega_{1} \wedge \cdots \wedge \omega_{q}=0, \quad 1 \leq i \leq q,
$$

of $\boldsymbol{F}$, for each element $[h]$ in $\operatorname{Ext}_{0}^{1}\left(\Omega_{F}, \mathcal{O}\right)$, we have the class $[h] c(d \omega)$ in $\mathscr{H}_{S}^{2}\left(\mathscr{O}^{q^{2}}\right)$ as defined in the previous section.

(6.1) THEOREM. If $\boldsymbol{F}$ is a codim $q$ reduced local foliation, the set $U(\boldsymbol{F})$ of equivalence classes of first order unfoldings of $\boldsymbol{F}$ is given by

$$
U(\boldsymbol{F})=\left\{[h] \in \operatorname{Ext}_{o}^{1}\left(\Omega_{F}, \mathcal{O}\right) \mid[h] c(d \omega)=0 \quad \text { in } \mathscr{H}_{S}^{2}\left(\mathcal{O}^{q^{2}}\right)\right\} .
$$

Proof. Let $U$ be a small polydisk with center 0 in $C^{n}$ on which the germs $\omega_{i}$ have representatives and let $F$ be the foliation on $U$ generated by $\omega_{1}, \cdots, \omega_{q}$. We set $S=S(F)$ and take a covering $\left\{U_{\lambda}\right\}_{\lambda \in A}$ of $U-S$ by small polydisks $U_{\lambda}$. If we write (see (5.12))

$$
d \omega_{i}=\sum_{j=1}^{q} \eta_{i j}^{\lambda} \wedge \omega_{j}, \quad 1 \leq i \leq q, \quad \text { on } U_{\lambda},
$$

then we have the identities (5.13) and (5.14). Now we take an element $[h]=\left[h_{1}, \cdots, h_{q}\right]$ in $\operatorname{Ext}_{0}^{1}\left(\Omega_{F}, \mathcal{O}\right)$. We may assume that the germs $h_{i}$ have representatives on $U$. Recall that the cohomology class $h c_{U}(\tau)$ is represented by the cocycle (5.15). 
Suppose that there exists a first order unfolding $\mathscr{F}^{(1)}$ of $\boldsymbol{F}$ which determines the system $h=\left(h_{1}, \cdots, h_{q}\right)$. Let $\mathscr{F}^{(1)}$ be a first order unfolding of $F$ on $U \times V$ which represents $\mathscr{F}^{(1)}$, where $V$ is a small disk with center 0 in $C=\{t\}$. We choose a system $\left(\tilde{\omega}_{1}, \cdots, \tilde{\omega}_{q}\right)$ of generators of $\mathscr{F}^{(1)}$ which extends the system $\left(\omega_{1}, \cdots, \omega_{q}\right)$. We may write

$$
\tilde{\omega}_{i} \equiv \omega_{i}+\omega_{i}^{(1)} t+h_{i} d t \quad \bmod \left(t^{2}, t d t\right), \quad 1 \leq i \leq q,
$$

where $\omega_{i}^{(1)}$ are 1-forms involving only $z$ and $d z$. Substituting (6.2) in the second identity of (4.7), we obtain

$$
\left(d h_{i}-\omega_{i}^{(1)}-\sum_{j=1}^{q} h_{j} \eta_{i j}^{\lambda}\right) \wedge \Omega=0 .
$$

Hence we may write

$$
d h_{i}-\omega_{i}^{(1)}-\sum_{j=1}^{q} h_{j} \eta_{i j}^{\lambda}=\sum_{k=1}^{q} \varphi_{i k}^{\lambda} \omega_{k}, \quad 1 \leq i \leq q, \quad \text { on } U_{\lambda},
$$

where $\varphi_{i k}^{\lambda}$ are holomorphic functions on $U_{\lambda}$. In the intersection $U_{\lambda} \cap U_{\mu}$, we have

$$
\sum_{j=1}^{q} h_{j} \psi_{i j}^{\lambda \mu} \omega_{k}=\sum_{j=1}^{q} h_{j} \eta_{i j}^{\lambda}-\sum_{j=1}^{q} h_{j} \eta_{i j}^{\mu}=\sum_{k=1}^{q}\left(\varphi_{i k}^{\mu}-\varphi_{i k}^{\lambda}\right) \omega_{k} .
$$

Since $\omega_{1}, \cdots, \omega_{q}$ are linearly independent at each point of $U_{\lambda} \cap U_{\mu}$, we get

$$
\sum_{j=1}^{q} h_{j} \psi_{i j k}^{\lambda \mu}=\varphi_{i k}^{\mu}-\varphi_{i k}^{\lambda}
$$

This shows that the cohomology class $h c_{U}(d \omega)$ is zero. Hence $[h] c(d \omega)=0$ in $\mathscr{H}_{S}^{2}\left(\mathcal{O}^{q^{2}}\right)$.

Conversely, if $[h] c(d \omega)=0$, then, by taking smaller $U$ and $U_{\lambda}^{\prime}$ s if necessary, there are functions $\varphi_{i k}^{\lambda}$ satisfying (6.4). If we set $\omega_{i}^{\lambda}=\sum_{k=1}^{q} h_{j} \eta_{i j}^{\lambda}+\sum_{k=1}^{q} \varphi_{i k}^{\lambda} \omega_{k}$ on $U_{\lambda}$, then it is easily checked that $\omega_{i}^{\lambda}=\omega_{i}^{\mu}$ on $U_{\lambda} \cap U_{\mu}$. Hence, for each $i, \omega_{i}^{\lambda}$ defines a 1 -form on $U-S$. By the assumption that $F$ is reduced, we see that codim $S \geq 2$ ((5.1) Lemma). Thus $\omega_{i}^{\lambda}$ extends to a 1 -form on $U$. We set

$$
\begin{aligned}
& \omega_{i}^{(1)}=d h_{i}-\sum_{j=1}^{q} h_{j} \eta_{i j}^{\lambda}-\sum_{k=1}^{q} \varphi_{i k}^{\lambda} \omega_{k} \quad \text { and } \\
& \tilde{\omega}_{i}=\omega_{i}+\omega_{i}^{(1)} t+h_{i} d t, \quad 1 \leq i \leq q .
\end{aligned}
$$

Then the system $\left(\tilde{\omega}_{i}, \cdots, \tilde{\omega}_{q}\right)$ satisfies the second identity of (4.7). We now show that the first is also satisfied. Using (6.5) we compute

$$
d \omega_{i}^{(1)} \wedge \Omega-d \omega_{i} \wedge\left(\sum_{j=1}^{q}(-1)^{j} \omega_{j}^{(1)} \wedge \Omega_{j}\right)=\sum_{j=1}^{q} h_{j}\left(\sum_{k=1}^{q} \eta_{i k}^{\lambda} \wedge \eta_{k j}^{\lambda}-d \eta_{i j}^{\lambda}\right) \wedge \Omega
$$


On the other hand, computing $d^{2} \omega_{i}$, we get

$$
\sum_{j=1}^{q}\left(d \eta_{i j}^{\lambda}-\sum_{k=1}^{q} \eta_{i k}^{\lambda} \wedge \eta_{k j}^{\lambda}\right) \wedge \omega_{j}=0
$$

Hence we have

$$
\left(d \eta_{i j}^{\lambda}-\sum_{k=1}^{q} \eta_{i k}^{\lambda} \wedge \eta_{k j}^{\lambda}\right) \wedge \Omega=(-1)^{j} \sum_{l=1}^{q}\left(d \eta_{i l}^{\lambda}-\sum_{k=1}^{q} \eta_{i k}^{\lambda} \wedge \eta_{k l}^{\lambda}\right) \wedge \omega_{l} \wedge \Omega_{j}=0 .
$$

Therefore, the system $\left(\tilde{\omega}_{1}, \cdots, \tilde{\omega}_{q}\right)$ satisfies the first order integrability condition. If we let $\mathscr{F}^{(1)}$ be the germ of the first order unfolding generated by $\left(\tilde{\omega}_{1}, \cdots, \tilde{\omega}_{q}\right)$, then $\mathscr{F}^{(1)}$ determines $h=\left(h_{1}, \cdots, h_{q}\right)$.

(6.7) Remark. By the above theorem, $U(F)$ is a sub-O-module, thus a sub-C-vector space, of $\operatorname{Ext}_{0}^{1}\left(\Omega_{F}, \mathcal{O}\right)$.

(6.8) REMARK. To find the first order unfoldings of a local foliation $F$,

$1^{\circ}$. Find $\operatorname{Ext}_{\sigma}^{1}\left(\Omega_{F}, \mathcal{O}\right)$ by (4.5).

$2^{\circ}$. Find $\eta_{i j}^{\lambda}$ and $\psi_{i j k}^{\lambda \mu}$ satisfying (6.2) and (5.13).

$3^{\circ}$. Pick up the elements $[h]=\left[h_{1}, \cdots, h_{q}\right]$ in $\operatorname{Ext}_{\odot}^{1}\left(\Omega_{F}, \mathcal{O}\right)$ such that (6.4) holds for some $\varphi_{i k}^{\lambda}$.

$4^{\circ}$. For each $[h]$ in $3^{\circ}$, determine $\tilde{\omega}$ by (6.5) and (6.6).

When the codimension of $\boldsymbol{F}$ is one, there is a simpler way. If we set $\omega=\omega_{1}$, then the first order integrability condition in (4.7) becomes

$$
\begin{aligned}
& d \omega^{(1)} \wedge \omega+\omega^{(1)} \wedge d \omega=0 \quad \text { and } \\
& h d \omega+\left(\omega^{(1)}-d h\right) \wedge \omega=0 .
\end{aligned}
$$

It is easy to show that (6.10) implies (6.9). If we use the cohomology group $H^{2}$ instead of $\mathscr{H}_{S}^{2}(\mathcal{O})((5.8))$, then the class $[h] c(d \omega)$ is represented by the form $h d \omega$. Hence we have ((6.1) Theorem)

$$
U(F)=\left\{[h] \in \operatorname{Ext}_{o}^{1}\left(\Omega_{F}, \mathcal{O}\right) \mid h d \omega=\eta \wedge \omega \text { for some } \eta \in \Omega\right\} .
$$

Thus if $[h]$ is in $U(F)$, and if $h d \omega=\eta \wedge \omega$,

$$
\tilde{\omega}=\omega+\omega^{(1)} t+h d t, \quad \omega^{(1)}=d h-\eta,
$$

is a first order unfolding representing $[h]$.

Note that if $\mathscr{F}$ is an unfolding of $\boldsymbol{F}$, then the image of the infinitesimal unfolding map of $\mathscr{F}$ is contained in $U(F)$.

(6.12) Definition. An element $[h]$ in $U(F)$ is said to be unobstructed if it is in the image of the infinitesimal unfolding map of a certain unfolding of $\boldsymbol{F}$, i.e., if the equivalence class $[h]$ is represented by an unfolding. 
If $\boldsymbol{F}=\left(d f_{1}, \cdots, d f_{q}\right)$ is a codim $q$ Haefliger foliation, then by (4.5),

$$
\operatorname{Ext}_{o}^{1}\left(\Omega_{F}, \mathcal{O}\right)=\mathcal{O}^{q} /\left(\frac{\partial f}{\partial z_{1}}, \cdots, \frac{\partial f}{\partial z_{n}}\right),
$$

where $\partial f / \partial \boldsymbol{z}_{j}$ denotes the vector $\left(\partial f_{1} / \partial \boldsymbol{z}_{j}, \cdots, \partial f_{q} / \partial \boldsymbol{z}_{j}\right)$. We have $U(F)=$ $\operatorname{Ext}_{\odot}^{1}\left(\Omega_{F}, \mathcal{O}\right)$ and none of the elements in $U(F)$ is obstructed. In fact, for any element $[h]=\left[h_{1}, \cdots, h_{q}\right]$ in $\operatorname{Ext}_{o}^{1}\left(\Omega_{F}, \mathcal{O}\right), \mathscr{F}=\left(d\left(f_{1}+h_{1} t\right), \cdots, d\left(f_{q}+h_{q} t\right)\right)$ is an unfolding of $F$ representing [h].

Also if $\mathscr{H}_{S}^{2}\left(\mathcal{O}^{q^{2}}\right)=0$, then we have $U(F)=\operatorname{Ext}_{0}^{1}\left(\Omega_{F}, \mathcal{O}\right)$. This is the case if codim $S(F) \geq 3$. In fact we have

(6.13) Theorem (Malgrange [6], [7], Moussu [8]). Let $\boldsymbol{F}$ be a codim $q$ local foliation. If codim $S(\boldsymbol{F}) \geq 3$, then $\boldsymbol{F}$ is a Haefliger foliation.

The proof of this theorem can be interpreted as a construction of a certain non-singular unfolding of $\boldsymbol{F}$. The most difficult part is the convergence of the formal construction. The result then follows from the Frobenius theorem.

It is not difficult to show the following

(6.14) Proposition. Let $\boldsymbol{F}=(\omega)$ be a codim 1 local foliation and let [1] denote the class in $\operatorname{Ext}_{0}^{1}\left(\Omega_{F}, \mathcal{O}\right)$ represented by the constant function 1. Then the following are equivalent:

(i ) $\boldsymbol{F}$ is a Haefliger foliation, i.e., $\omega=$ gdf for some $f$ in $\mathcal{O}$ and a unit $g$ in $\mathcal{O}$.

(ii) $U(F)=\operatorname{Ext}_{0}^{1}\left(\Omega_{F}, \mathcal{O}\right)$ and none of the elements in $U(F)$ is obstructed.

(iii) [1] is in $U(\boldsymbol{F})$ and is not obstructed.

(6.15) Definition. An unfolding $\mathscr{F}$ of a local foliation $\boldsymbol{F}$ is infinitesimally versal if the image of the infinitesimal unfolding map of $\mathscr{F}$ is $U(F)$.

In [10], it is proved that an infinitesimally versal unfolding of a codim 1 local (reduced) foliation is versal.

(6.16) Definition. An unfolding $\mathscr{F}$ of $F$ is universal if it is versal and if the infinitesimal unfolding map of $\mathscr{F}$ is injective.

(6.17) Examples. Let $n=2, q=1$ and let $\boldsymbol{F}=(\omega)$ be given by

$1^{\circ}$. $\omega=z_{2} d z_{1}-z_{1} d z_{2}$.

We have $\operatorname{Ext}_{0}^{1}\left(\Omega_{F}, \mathcal{O}\right)=C\left\{z_{1}, z_{2}\right\} /\left(z_{1}, z_{2}\right)=C$ and we may take [1] as a basis element. Since $d \omega=-2 d z_{1} \wedge d z_{2}$, we see that $U(F)=0$. Hence by the versality theorem in [10], $\boldsymbol{F}$ is a universal unfolding of $\boldsymbol{F}$ itself and every unfolding of $F$ is trivial. We remark that if we try to construct an unfolding of $\boldsymbol{F}$ representing [1], we obtain a "meromorphic" foliation. Namely, since 


$$
d \omega=\eta \wedge \omega, \quad \eta=\frac{2}{z_{1}} d z_{1}
$$

setting $\omega^{(1)}=d h-\eta=-\left(2 / z_{1}\right) d z_{1}$, we have

$$
\tilde{\omega}=\omega+\omega^{(1)} t+h d t=\left(z_{2}-\frac{2}{z_{1}} t\right) d z_{1}-z_{1} d z_{2}+d t .
$$

It is easily shown that $\tilde{\omega}$ actually satisfies the integrability $d \tilde{\omega} \wedge \tilde{\omega}=0$.

$2^{\circ}$. $\omega=z_{2}^{2} d z_{1}-z_{1} d z_{2}$.

We have $\operatorname{Ext}_{\odot}^{1}\left(\Omega_{F}, \mathcal{O}\right)=C\left\{z_{1}, z_{2}\right\} /\left(z_{1}, z_{2}^{2}\right)=C^{2}$. However, it is easily checked that $U(F)=0$.

$3^{\circ}$. $\omega=z_{2}^{2} d z_{1}-z_{1}^{2} d z_{2}$.

We have $\operatorname{Ext}_{\odot}^{1}\left(\Omega_{F}, \mathcal{O}\right)=C^{4}$ and we may take [1], $\left[z_{1}\right],\left[z_{2}\right]$ and $\left[z_{1} z_{2}\right]$ as basis elements. Also it is not difficult to see that $U(F)=C^{2}$ and we may take $\left[z_{1}-z_{2}\right]$ and $\left[z_{1} z_{2}\right]$ as basis elements. If we set $h_{1}=z_{1} z_{2}$, then we have

$$
h_{1} d \omega=\eta_{1} \wedge \omega, \quad \eta_{1}=2 d\left(z_{1} z_{2}\right) .
$$

Thus $\mathscr{F}_{1}=\left(\tilde{\omega}_{1}\right)$,

$$
\tilde{\omega}_{1}=\omega+\omega_{1}^{(1)} t_{1}+h_{1} d t_{1}, \quad \omega_{1}^{(1)}=d h_{1}-\eta_{1}=-d\left(z_{1} z_{2}\right),
$$

is a first order unfolding of $F$ representing $h_{1}=z_{1} z_{2}$. Moreover, it is easily checked that $\tilde{\omega}_{1}$ is integrable; $d \tilde{\omega}_{1} \wedge \tilde{\omega}_{1}=0$, and thus $\mathscr{F}_{1}$ is actually an unfolding of $F$. Also if we set $h_{2}=z_{1}-z_{2}$, we have

$$
h_{2} d \omega=\eta_{2} \wedge \omega, \quad \eta_{2}=2 d\left(z_{1}-z_{2}\right) \text {. }
$$

Thus $\mathscr{F}_{2}=\left(\tilde{\omega}_{2}\right)$,

$$
\tilde{\omega}_{2}=\omega+\omega_{2}^{(1)} t_{2}+h_{2} d t_{2}, \quad \omega_{2}^{(1)}=d h_{2}-\eta_{2}=-d\left(z_{1}-z_{2}\right),
$$

is a first order unfolding of $\boldsymbol{F}$ representing $h_{2}=z_{1}-z_{2}$. It is easily checked that $\tilde{\omega}_{2}$ is integrable and $\mathscr{F}_{2}$ is also an unfolding of $\boldsymbol{F}$. We combine $\tilde{\omega}_{1}$ and $\tilde{\omega}_{2}$ to obtain an integrable form

$$
\begin{aligned}
\tilde{\omega} & =\omega+\omega_{1}^{(1)} t_{1}+\omega_{2}^{(1)} t_{2}+h_{1} d t_{1}+h_{2} d t_{2}-t_{2} d t_{1}+t_{1} d t_{2} \\
& =\left(z_{2}^{2}-z_{2} t_{1}-t_{2}\right) d z_{1}-\left(z_{1}^{2}+z_{1} t_{1}-t_{2}\right) d z_{2}+\left(z_{1} z_{2}-t_{2}\right) d t_{1}+\left(z_{1}-z_{2}+t_{1}\right) d t_{2} .
\end{aligned}
$$

Then by the versality theorem in [10], $\mathscr{F}=(\tilde{\omega})$ is a universal unfolding of $\boldsymbol{F}$.

$4^{\circ} . \omega=z_{2} d z_{1}+\left(z_{1}+z_{1}^{2} z_{2}\right) d z_{2}$.

We have $\operatorname{Ext}_{\odot}^{1}\left(\Omega_{F}, \mathcal{O}\right)=C\left\{z_{1}, z_{2}\right\} /\left(z_{1}, z_{2}\right)=C$. Since

$$
d \omega=\eta \wedge \omega, \quad \eta=-2 z_{1} d z_{2},
$$


we have $U(F)=\operatorname{Ext}_{\mathscr{\odot}}^{1}\left(\Omega_{F}, \mathcal{O}\right)$ and $\mathscr{F}^{(1)}=(\tilde{\omega})$,

$$
\tilde{\omega}=z_{2} d z_{1}+\left(z_{1}+z_{1}^{2} z_{2}+2 z_{1} t\right) d z_{2}+d t
$$

is a first order unfolding representing [1]. However, since $\omega$ does not admit integrating factors, $\mathscr{F}^{(1)}$ is not equivalent to an unfolding. Thus the element [1] (and hence every non-zero element) in $U(F)$ is obstructed.

For more examples and applications, see [11], [14] and [15].

\section{§ 7. First order unfoldings of global foliations}

Let $M$ be a compact complex manifold. If we denote by $D(M)$ the set of equivalence classes of first order deformations of $M$, then it is well-known that there is a bijection

$$
D(M) \simeq H^{1}\left(M, \Theta_{M}\right)
$$

(7.2) REMARK. If $\mathscr{M}^{(1)}$ is a first order deformation of $M$, then it is represented by the following data:

(i) a finite open covering $\left\{U_{\lambda}\right\}_{\lambda \in A}$ of $M$ by coordinate neighborhoods $U_{\lambda}$ (If we denote by $z^{\lambda}=\left(z_{1}^{\lambda}, \cdots, z_{n}^{\lambda}\right)$ a coordinate system on $U_{\lambda}$, then in the intersection $U_{\lambda} \cap U_{\mu}, z^{\lambda}$ is a vector-valued holomorphic function of $z^{\mu} ; z^{\lambda}=$ $\left.f^{\lambda \mu}\left(z^{\mu}\right)\right)$

(ii) a neighborhood $V$ of 0 in $C=\{t\}$ and

(iii) a collection $\left\{\Psi_{\lambda \mu}\right\}$, where for each pair $(\lambda, \mu), \Psi_{\lambda \mu}$ is a biholomorphic map of $\left(U_{\lambda} \cap U_{\mu}\right) \times V$ onto itself of the form $\Psi_{\lambda \mu}\left(z^{\mu}, t\right)=\left(f^{\lambda \mu}\left(z^{\mu}, t\right), t\right)$, satisfying

$f^{\lambda \mu}\left(z^{\mu}, 0\right)=f^{\lambda \mu}\left(z^{\mu}\right)$

$f^{\lambda \nu}\left(z^{\nu}, t\right) \equiv f^{\lambda \mu}\left(f^{\mu \nu}\left(z^{\nu}, t\right), t\right), \quad$ for $\left(z^{\lambda}, t\right)$ in $\left(U_{\lambda} \cap U_{\mu} \cap U_{\nu}\right) \times V, \quad \bmod t^{2}$.

Under the bijection (7.1), the equivalence class of $\mathscr{M}^{(1)}$ corresponds to the cohomology class represented by the cocycle $\left\{\sum_{i=1}^{n} \frac{f_{i}^{\lambda \mu}}{\partial t}\left(z^{\mu}, 0\right) \frac{\partial}{\partial z_{i}^{\lambda}}\right\}$.

Now let $F$ be a codim $q$ foliation (of complete intersection type) on $M$.

(7.3) Definition. A first order unfolding $\mathscr{F}^{(1)}$ of $F$ consists of

(i ) a first order deformation $\mathscr{M}^{(1)}=\left(\left\{U_{\lambda}\right\}, V,\left\{\Psi_{\lambda \mu}\right\}\right)$ of $M$,

(ii) a collection $\left\{\mathscr{F}_{i}^{(1)}\right\}$, where, for each $\lambda, \mathscr{F}_{\lambda}^{(1)}$ is a first order unfolding of $\left.F\right|_{U_{\lambda}}$ on $U_{\lambda} \times V$, satisfying

$$
\left.\mathscr{F}_{\mu}^{(1)}\right|_{\left(U_{\left.\lambda \cap U_{\mu}\right) \times V}\right.} \equiv\left(\Psi_{\lambda \mu}\right) * *\left(\left.\mathscr{F}_{\lambda}^{(1)}\right|_{\left(U_{\left.\lambda \cap U_{\mu}\right) \times V}\right)} \bmod \left(t^{2}, t d t\right) .\right.
$$

The equivalence between two first order unfoldings of $(M, F)$ is defined in an obvious way. Let $U(M, F)$ denote the set of equivalence classes of 
first order unfoldings of $(M, F)$. Then we have an embedding

$$
U(M, F) G \operatorname{Ext}_{\mathscr{O}_{M}}^{1}\left(\Omega_{F}, \mathcal{O}_{M}\right),
$$

which assings to the equivalence class of a first order unfolding $\left(\mathscr{M}^{(1)}, \mathscr{F}^{(1)}\right)$ $=\left(\left\{U_{\lambda}\right\}, V,\left\{\Psi^{\lambda \mu}\right\},\left\{\mathscr{F}_{\lambda}^{(1)}\right\}\right)$, the class in $\operatorname{Ext}_{\mathscr{O}_{M}}^{1}\left(\Omega_{F}, \mathcal{O}_{M}\right)$ (see (3.3)) represented by $(\theta, \varphi)=\left(\left\{\theta^{\lambda \mu}\right\},\left\{\varphi^{\lambda}\right\}\right)$ with

$$
\theta^{\lambda \mu}=\sum_{i=1}^{n} \frac{\partial f_{i}}{\partial t}\left(z^{\mu}, 0\right) \frac{\partial}{\partial z_{i}^{\lambda}} \quad \text { and } \quad \varphi^{\lambda}(\omega)=h_{\lambda},
$$

where for a 1-form $\omega$ in $\Gamma\left(U_{\lambda}, F\right), h_{\lambda}$ is the holomorphic function on $U_{\lambda}$ given as the coefficient of $d t$ of an extension $\tilde{\omega}$ of $\omega$ in $\Gamma\left(U_{\lambda} \times V, \mathscr{F}_{\lambda}^{(1)}\right)$.

Now we determine the set $U(M, F)$. Recall that for each point $z$ in $M$, the set $U\left(F_{z}\right)$ of equivalence classes of first order unfoldings of the germ $F_{z}$ of the foliation $F$ at $z$ is canonically embedded in $\operatorname{Ext}_{O_{M, z}}^{1}\left(\Omega_{F, z}, \mathcal{O}_{M, z}\right)=\mathscr{E} x t_{O_{M}}^{1}\left(\Omega_{F}, \mathcal{O}_{M}\right)_{z}$ (section 4) and the set is determined in section 6. We define $\mathscr{U}(M, F)$ to be the subsheaf of $\mathscr{E}_{x t_{O_{M}}^{1}}\left(\Omega_{F}, \mathcal{O}_{M}\right)$ given by $\mathscr{U}(M, F)=\bigcup_{z \in M} U\left(F_{z}\right)$ and call it the sheaf of germs of first order unfoldings of $(M, F)$.

Let $\varepsilon$ be the edge homomorphism in (2.10). The proof of the following theorem is straightforward.

(7.4) Theorem. The set $U(M, F)$ of equivalence classes of first order unfoldings of $(M, F)$ is given by

$$
U(M, F)=\varepsilon^{-1}(\Gamma(M, \mathscr{U}(M, F))) .
$$

\section{References}

[1] P. Baum, Structure of foliation singularities, Advances in Math., 15 (1975), 361-374.

[2] P. Baum and R. Bott, Singularities of holomorphic foliations, J. Differential Geometry, 7 (1972), 279-342.

[ 3 ] A. Grothendieck, Local Cohomology (notes by R. Hartshorne), Lecture Notes in Math. 41, Springer-Verlag, Heidelberg, 1967.

[4] E. Horikawa, On deformations of holomorphic maps, I and II, J. Math. Soc. Japan, 25 (1973), 372-396, ibid, 26 (1974), 647-667.

[5] K. Kodaira and D. C. Spencer, On deformations of complex analytic structures, I and II, Ann. of Math., 67 (1958), 328-466.

[6] B. Malgrange, Frobenius avec singularités, 1. Codimension un, Publ. Math. I.H.E.S., 46 (1976), 163-173.

[ 7 ] B. Malgrange, Frobenius avec singularités, 2. Le cas général, Invent. Math., 39 (1977), 67-89.

[8] R. Moussu, Sur l'existence d'intégrales premières pour un germe de forme de Pfaff, Ann. Inst. Fourier, 26 (1976), 171-220.

[9] K. Saito, On a generalization of de Rham lemma, Ann. Inst. Fourier, 26 (1976), 165-170. 
[10] T. Suwa, A theorem of versality for unfoldings of complex analytic foliation singularities, Invent. Math., 65 (1981), 29-48.

[11] T. Suwa, Kupka-Reeb phenomena and universal unfoldings of certain foliation singularities, to appear in Osaka J. Math. .

[12] T. Suwa, Residues of complex analytic foliation singularities, to appear in J. Math. Soc. Japan.

[13] T. Suwa, Singularities of complex analytic foliations, to appear in the Proceedings of Symposia in Pure Math. 40, Amer. Math. Soc. .

[14] T. Suwa, Unfoldings of meromorphic functions, Math. Ann., 262 (1983), 215-224.

[15] T. Suwa, Unfoldings of foliations with multiform first integrals, to appear in Ann. Inst. Fourier, 33 (1983).

[16] R. Thom, On singularities of foliations, Manifolds-Tokyo 1973, edited by A. Hattori, Univ. of Tokyo Press, Tokyo 1975, 171-173.

[17] R. Thom, Limit set of leaves of analytic foliations (Japanese translation by T. Fukuda of an invited address presented at the 100th anniversary of the Math. Soc. of Japan), Sugaku 30, Iwanami Shoten (1978), 132-136.

\section{DePaRTMENT OF Mathematic \\ HOKKAIDO UNIVERSITY \\ SAPPORO 060, JAPAN}

